\title{
Optimal control for two-dimensional stochastic second grade fluids
}

\author{
Nikolai Chemetov1, Fernanda Cipriand 2
}

\begin{abstract}
This article deals with a stochastic control problem for certain fluids of non-Newtonian type. More precisely, the state equation is given by the two-dimensional stochastic second grade fluids perturbed by a multiplicative white noise. The control acts through an external stochastic force and we search for a control that minimizes a cost functional. We show that the Gâteaux derivative of the control to state map is a stochastic process being the unique solution of the stochastic linearized state equation. The well-posedness of the corresponding stochastic backward adjoint equation is also established, allowing to derive the first order optimality condition.
\end{abstract}

Key words. Stochastic second grade fluids, Backward stochastic partial differential equations, Stochastic optimal control, Necessary optimality condition.

AMS Subject Classification. 35R60, 49K20, 60G15, 60H15, 76D55.

\section{Introduction}

This paper is devoted to the study of a control problem for stochastic incompressible fluid of second grade filling a bounded two-dimensional domain $\mathcal{O}$. The evolution equations are given by

$$
\left\{\begin{array}{lr}
\frac{\partial}{\partial t}(Y-\alpha \Delta Y)=\nu \Delta Y-\operatorname{curl}(Y-\alpha \Delta Y) \times Y-\nabla \pi+U+G(t, Y) \dot{W}_{t} \\
\operatorname{div} Y=0 & \text { in }(0, T) \times \mathcal{O},
\end{array}\right.
$$

where $Y$ denotes the velocity of the fluid, $G(t, Y) \dot{W}_{t}$ is a multiplicative white noise and the control is exercised through an adapted stochastic process $U=U(\omega, t)$. Due to the adaptiveness, such stochastic external force $U$ also acts as a feedback control in the sense of the noise.

To study the well-posedness of the state equations (1.1), we should impose a suitable boundary condition on the boundary $\Gamma$ of the domain $\mathcal{O}$. Considering the classical homogeneous Dirichlet boundary condition, the authors in [26] proved the existence and uniqueness of strong stochastic solutions. Other physically relevant boundary condition is the so-called Navier-slip boundary condition, which reads as

$$
Y \cdot \mathrm{n}=0, \quad(\mathrm{n} \cdot D Y) \cdot \tau=0 \quad \text { on } \Gamma,
$$

\footnotetext{
${ }^{1}$ Departamento de Matemática, Faculdade de Ciências da Universidade de Lisboa. E-mail: nvchemetov@fc.ul.pt.

${ }^{2}$ Centro de Matemática e Aplicações (CMA) FCT/UNL and Departamento de Matemática, Faculdade de Ciências e Tecnologia da Universidade Nova de Lisboa. E-mail: cipriano@fct.unl.pt.
} 
where $\mathrm{n}=\left(n_{1}, n_{2}\right)$ and $\tau=\left(-n_{2}, n_{1}\right)$ are the unit normal and tangent vectors, respectively, to the boundary $\Gamma$ and $D Y=\frac{1}{2}\left[\nabla Y+\nabla Y^{\top}\right]$ is the rate-of-strain tensor. This boundary condition allows fluid particles to move tangentially to the boundary of the domain and for the NavierStokes fluid it has the special feature of being compatible with the limit transition on vanishing viscosity (see [7, [8], [12, 13, 21]). Let us refer that the state equations (1.1) supplemented with the Navier-slip boundary condition (1.2) has been studied in the article [9, where the authors obtained the existence and uniqueness results, as well as the stability properties of the solutions. Concerning the physical justification and relevance of the second grade fluid equations, we refer to the articles [17, [18, 25] and [27. Related non-viscous model can be found in [19] and [20].

The main goal of the present article is to study the control problem for the stochastic state equation (1.1) with the Navier-slip boundary condition (1.2). As far as we know this is the first article where the control problem is addressed for the stochastic second grade fluids. Let us mention the three key steps to be overtaken in solving the control problem: one of them relies on the study of the Gâteau derivative of the control to state map, which will be given by the unique solution of the stochastic linearized state equation, the second one consists in the determination and well-posedness of the stochastic backward adjoint equations in a suitable functional space, and the third one is the relation between the solution of the linearized equation and the adjoint state solution, which allows to write the necessary first order optimality condition in terms of the adjoint state.

In the framework of the Newtonian fluids, we refer [14, [15, 24], 29] where the authors studied control problems for the stochastic Navier-Stokes involving different techniques. In the present work we follow the methods introduced in [4, 22] and 23] for the two-dimensional NavierStokes equations. When comparing with the Navier-Stokes equations, here the main difficulty relies on the fact that the nonlinear term contains third order derivatives that substantially complicates the analysis. Fortunately, we take advantage of the Navier-slip boundary condition because it allows to estimate the vorticity of the fluid on the boundary throughout the tangential velocity which roughly speaking reduces the order of the derivatives, and consequently allows to obtain a priori estimates for the Galerkin approximations of the forward stochastic linearized and backward stochastic adjoint equations in the Sobolev space $H^{2}$.

The plan of the present paper is as follows. The formulation of the problem is stated in Section 2. In Section 3, we present some auxiliary lemmas concerning the normas of the functional spaces, relevant properties and estimates of the nonlinear terms. In Section 4, we collect useful results and estimates for the solution of the stochastic state equation. Section 5 deals with the wellposedness of the stochastic linearized equation. In Section 6, we analyse the derivative of the control to state map. Section 7 is devoted to the formulation of the stochastic backward adjoint equations and to the study of the existence and uniqueness of the solutions. We also improve the integrability properties for the solution of the stochastic state equation, and deduce the duality relation between the linearized and the adjoint stochastic processes. Finally, in Section 8 we establish the main result of the article, by proving the existence of a solution to the control problem and establishing a first order optimality condition. 


\section{Functional spaces and formulation of the problem}

We consider a stochastic second grade fluid model in a bounded domain $\mathcal{O}$ of $\mathbb{R}^{2}$ with a sufficiently regular boundary $\Gamma$

$$
\begin{cases}d(\sigma(Y))=(\nu \Delta Y-\operatorname{curl}(\sigma(Y)) \times Y-\nabla \pi+U) d t+G(t, Y) d W_{t}, & \\ \operatorname{div} Y=0 & \text { in }(0, T) \times \mathcal{O}, \\ Y \cdot \mathrm{n}=0, \quad(\mathrm{n} \cdot D Y) \cdot \tau=0 & \text { on }(0, T) \times \Gamma, \\ Y(0)=Y_{0} & \text { in } \mathcal{O},\end{cases}
$$

where $\nu>0$ is a constant viscosity of the fluid, $\alpha>0$ is a constant material modulus, $\Delta$ and $\nabla$ denote the Laplacian and the gradient, $Y=\left(Y_{1}, Y_{2}\right)$ is a $2 \mathrm{D}$ velocity field and

$$
\sigma(Y)=Y-\alpha \Delta Y
$$

The function $\pi$ represents the pressure, $U$ is a distributed mechanical force and the term $G(t$, $Y) d W_{t}$ corresponds to the stochastic perturbation, where $W_{t}=\left(W_{t}^{1}, \ldots, W_{t}^{m}\right)$ is a standard $\mathbb{R}^{m}$-valued Wiener process defined on a complete probability space $(\Omega, \mathcal{F}, P)$ endowed with a filtration $\left\{\mathcal{F}_{t}\right\}_{t \in[0, T]}$ for the Wiener process.

Let us define some normed spaces. Let $X$ be a real Banach space with norm $\|\cdot\|_{X}$. We denote by $L^{p}(0, T ; X)$ the space of $X$-valued measurable $p$-integrable functions defined on $[0, T]$ for $p \geq 1$.

For $p, r \geq 1$ let $L^{p}\left(\Omega, L^{r}(0, T ; X)\right)$ be the space of stochastic processes $Y=Y(\omega, t)$ with values in $X$ defined on $\Omega \times[0, T]$, adapted to the filtration $\left\{\mathcal{F}_{t}\right\}_{t \in[0, T]}$, and endowed with the norms

$$
\|Y\|_{L^{p}\left(\Omega, L^{r}(0, T ; X)\right)}=\left(\mathbb{E}\left(\int_{0}^{T}\|Y\|_{X}^{r} d t\right)^{\frac{p}{r}}\right)^{\frac{1}{p}}
$$

and

$$
\|Y\|_{L^{p}\left(\Omega, L^{\infty}(0, T ; X)\right)}=\left(\mathbb{E} \sup _{t \in[0, T]}\|Y\|_{X}^{p}\right)^{\frac{1}{p}} \quad \text { if } r=\infty,
$$

where $\mathbb{E}$ is the mathematical expectation with respect to the probability measure $P$. As usual in the notation of processes $Y=Y(\omega, t)$ we normally omit the dependence on $\omega \in \Omega$.

In equation (2.1) the vector product $\times$ for $2 \mathrm{D}$ vectors $u=\left(u_{1}, u_{2}\right)$ and $v=\left(v_{1}, v_{2}\right)$ is calculated as $u \times v=\left(u_{1}, u_{2}, 0\right) \times\left(v_{1}, v_{2}, 0\right)$; the curl of the vector $u$ is equal to curl $u=\frac{\partial u_{2}}{\partial x_{1}}-\frac{\partial u_{1}}{\partial x_{2}}$ and the vector product of $\operatorname{curl} u$ with the vector $v$ is understood as

$$
\operatorname{curl} u \times v=(0,0, \operatorname{curl} u) \times\left(v_{1}, v_{2}, 0\right) .
$$

Given two vectors $u, v \in \mathbb{R}^{2}, u \cdot v=\sum_{i=1}^{2} u_{i} v_{i}$ corresponds to the usual scalar product in $\mathbb{R}^{2}$.

Let us introduce the following Hilbert spaces

$$
\begin{aligned}
H & =\left\{v \in L^{2}(\mathcal{O}) \mid \operatorname{div} v=0 \text { in } \mathcal{O} \text { and } v \cdot \mathrm{n}=0 \text { on } \Gamma\right\}, \\
V & =\left\{v \in H^{1}(\mathcal{O}) \mid \operatorname{div} v=0 \text { in } \mathcal{O} \text { and } v \cdot \mathrm{n}=0 \text { on } \Gamma\right\}, \\
W & =\left\{v \in V \cap H^{2}(\mathcal{O}) \mid(\mathrm{n} \cdot D v) \cdot \tau=0 \text { on } \Gamma\right\} \\
\widetilde{W} & =W \cap H^{3}(\mathcal{O}) .
\end{aligned}
$$

We denote by $(\cdot, \cdot)$ the inner product in $L^{2}(\mathcal{O})$ and by $\|\cdot\|_{2}$ the associated norm. The norm in the space $H^{p}(\mathcal{O})$ is denoted by $\|\cdot\|_{H^{p}}$. 
Let us consider the Helmholtz projector $\mathbb{P}: L^{2}(\mathcal{O}) \longrightarrow H$. It is well know that $\mathbb{P}$ is a linear bounded operator being characterized by the equality $\mathbb{P} v=\tilde{v}$, where $\tilde{v}$ is defined by the Helmholtz decomposition

$$
v=\tilde{v}+\nabla \phi, \quad \tilde{v} \in H \quad \text { and } \quad \phi \in H^{1}(\mathcal{O}) .
$$

On the functional spaces $V, W$ and $\widetilde{W}$ defined in (2.2), we introduce the inner products

$$
\begin{aligned}
(u, v)_{V} & =(\sigma(u), v)=(u, v)+2 \alpha(D u, D v), \\
(u, v)_{W} & =(\mathbb{P} \sigma(u), \mathbb{P} \sigma(v))+(u, v)_{V}, \\
(u, v)_{\widetilde{W}} & =(\operatorname{curl} \sigma(u), \operatorname{curl} \sigma(v))+(u, v)_{V}
\end{aligned}
$$

and denote by $\|\cdot\|_{V},\|\cdot\|_{W},\|\cdot\|_{\widetilde{W}}$ the corresponding norms. Let us notice that the functional spaces $V, W$ and $\widetilde{W}$ defined in (2.2) being subspaces of Sobolev spaces are naturally endowed with the Sobolev norms, however it is more convenient to use the norms induced by the inner products (2.3) that are related with the structure of the equations.

The main goal of this paper is to control the solution of the stochastic model (2.1) by the stochastic distributed mechanical force $U$, which belongs to a suitable admissible set $\mathcal{U}_{a d}^{b}$, that will be defined later on.

Let us consider the cost functional

$$
J\left(U, Y_{U}\right)=\mathbb{E} \int_{0}^{T} L\left(t, U, Y_{U}\right) d t+\mathbb{E} h\left(Y_{U}(T)\right), \quad U \in \mathcal{U}_{a d}^{b},
$$

which will be specified in Section 8 . We aim to control the solution $Y$ of the stochastic second grade fluid equations (2.1), minimizing the cost functional (2.4) for an appropriate force $U \in \mathcal{U}_{a d}^{b}$. More precisely, our purpose is to solve the following problem

$$
(\mathcal{P})\left\{\begin{aligned}
\underset{U}{\operatorname{minimize}}\{J(U, Y) & : U \in \mathcal{U}_{a d}^{b} \text { and } \\
Y & \text { is the solution of system (2.1) for the minimizing } \left.U \in \mathcal{U}_{a d}^{b}\right\} .
\end{aligned}\right.
$$

Remark 2.1 A standard example of the cost functional 2.4 is presented as

$$
J\left(U, Y_{U}\right)=\mathbb{E} \int_{0}^{T} \int_{\mathcal{O}}\left|Y_{U}-Y_{d}\right|^{2} d x d t+\lambda \mathbb{E} \int_{0}^{T} \int_{\mathcal{O}}|U|^{2} d x d t+\mathbb{E} h\left(Y_{U}(T)\right)
$$

with a desired target field $Y_{d} \in L^{2}(\Omega \times(0, T) \times \mathcal{O})$ and some given $\lambda \geq 0$. Hence the Lagrangian $L$ is defined by

$$
L\left(t, U, Y_{U}\right)=\left\|Y_{U}-Y_{d}\right\|_{2}^{2}+\frac{\lambda}{2}\|U\|_{2}^{2} .
$$

\section{Preliminaries}

In this section, we present some useful lemmas concerning the properties of the operators that will be involved in the study of the stochastic forward and backward differential equations that 
will be analyzed in the next sections. They are straightforward adaptations of analogous results in the literature, however, for the convenience of the reader, we present the sketch of some proofs.

The first lemma is related with the boundary condition yielding a characterization of the Navier slip boundary condition (1.2) for the velocity field in terms of its vorticity (see Lemma 4.1 and Corollary 4.2 in [21]). Denoting by $k$ the curvature of $\Gamma$ and parameterizing $\Gamma$ by arc length $s$, we have

$$
\frac{\partial \mathrm{n}}{\partial \tau}=\frac{d \mathrm{n}}{d s}=k \tau
$$

Let us recall the following relation between the anti-symmetric tensor $A v=\nabla v-(\nabla v)^{\top}$ and the vorticity operator:

$$
A v=\operatorname{curl} v\left[\begin{array}{rr}
0 & -1 \\
1 & 0
\end{array}\right]
$$

Lemma 3.1 Let $v \in H^{2}(\mathcal{O})$ be a vector field fulfilling the Navier slip boundary condition (1.2). Then

$$
\operatorname{curl} v=g(v) \quad \text { with } \quad g(v)=2 k v \cdot \tau \quad \text { on } \Gamma \text {. }
$$

Proof. The symmetry of $D v$ and the anti-symmetry of $A v$ imply that

$$
(D v) \tau \cdot \mathrm{n}=(D v) \mathrm{n} \cdot \tau \quad \text { and } \quad(A v) \tau \cdot \mathrm{n}=-(A v) \mathrm{n} \cdot \tau .
$$

It follows that $(\nabla v) \tau \cdot \mathrm{n}=(D v) \mathrm{n} \cdot \tau-\frac{1}{2}(A v) \mathrm{n} \cdot \tau$, which is equivalent to

$$
\operatorname{curl} v=-2(\nabla v) \tau \cdot \mathrm{n}+2(D v) \mathrm{n} \cdot \tau \text {. }
$$

Taking the derivative of the expression $v \cdot \mathrm{n}=0$ in the direction of the tangent vector $\tau$, we deduce

$$
(\nabla v) \tau \cdot \mathrm{n}=-k v \cdot \tau
$$

The conclusion is then a consequence of (3.2) and (3.3).

The next result follows from Lemma 5 in [5], and it allows to deduce uniform estimates for the Galerkin approximations of the linearized and adjoint equations in the Sobolev space $H^{2}$.

Lemma 3.2 For each $v \in W$, we have

$$
\begin{gathered}
\|\sigma(v)-\mathbb{P} \sigma(v)\|_{2} \leq C\|v\|_{H^{1}}, \\
\|\sigma(v)-\mathbb{P} \sigma(v)\|_{H^{1}} \leq C\|v\|_{H^{2}} .
\end{gathered}
$$

In the above lemma and throughout the article, we denote by $C$ a generic positive constant that can take different values, and may depend only on the domain $\mathcal{O}$, the regularity of the boundary $\Gamma$ and the physical constant $\alpha$.

The following result is a direct consequence of the Korn inequality.

Lemma 3.3 There exist some positive constants $C_{*}, K_{*}$, such that

$$
\begin{gathered}
\|u\|_{H^{1}}^{2} \leq C_{*}\|u\|_{V}^{2}, \quad \forall u \in V, \\
\|u\|_{H^{1}}^{2} \leq K_{*}\|D u\|_{2}^{2}, \quad \forall u \in V,
\end{gathered}
$$


Proof. The first inequality is a consequence of the Korn inequality

$$
\|u\|_{H^{1}} \leq C\left(\|D u\|_{2}+\|u\|_{2}\right), \quad \forall u \in H^{1}(\mathcal{O})
$$

where $C$ is a positive constant just depending on $\mathcal{O}$. Taking into account the results in [16], for non axisymmetric domains, we also have (3.7).

Now, we introduce the modified Stokes problem with the Navier-slip boundary condition

$$
\begin{cases}h-\alpha \Delta h+\nabla p=f, \quad \operatorname{div} h=0 & \text { in } \mathcal{O}, \\ h \cdot \mathrm{n}=0, \quad(\mathrm{n} \cdot D h) \cdot \tau=0 & \text { on } \Gamma\end{cases}
$$

that according to [28, for any $f \in H^{m}(\mathcal{O}), m \in \mathbb{N}$, has a unique solution $(h, p)$ which belongs to $H^{m+2}(\mathcal{O}) \times H^{m+1}(\mathcal{O})$ and verifies

$$
\|h\|_{H^{m+2}} \leq C\|f-h\|_{H^{m}} .
$$

In what follows, we also denote the solution $h$ as

$$
h=(I-\alpha \Delta)^{-1} f .
$$

Next we state a lemma concerning the equivalence of the norms, see Propositions 3 in [6] and Lemma 2.1 in 10 for similar results.

Lemma 3.4 There exist some positive constants $C, C_{* *}$, such that

$$
\begin{array}{cc}
\|u\|_{H^{2}}^{2} \leq C\|u\|_{W}^{2}, & \forall u \in W, \\
\|u\|_{H^{3}}^{2} \leq C\|u\|_{\widetilde{W}}^{2}, & \forall u \in \widetilde{W}, \\
C_{* *}\|u\|_{\widetilde{W}}^{2} \leq\|u\|_{* *}^{2}, & \forall u \in \widetilde{W},
\end{array}
$$

where $\|u\|_{* *}^{2}=2 \alpha\|D u\|_{2}^{2}+\|\operatorname{curl} \sigma(u)\|_{2}^{2}$.

Proof. 1) Multiplying (3.8) 1 by $h$, we derive

$$
\|h\|_{2}^{2}+2 \alpha\|D h\|_{2}^{2}=(f, h) \leq\|f\|_{2}\|h\|_{2},
$$

that is

$$
\|h\|_{2} \leq\|f\|_{2}, \quad\|D h\|_{2} \leq C\|f\|_{2} .
$$

Due to (3.9) we have

$$
\|h\|_{H^{2}} \leq C\|f-h\|_{2} \leq C\left(\|f\|_{2}+\|h\|_{2}\right) \leq C\|f\|_{2} .
$$

Hence taking $f=\mathbb{P} \sigma(u)$ in (3.8) 1 and using (3.13), we get

$$
\|h\|_{H^{2}} \leq C\|\mathbb{P} \sigma(u)\|_{2} \text {. }
$$

By another hand if we use estimates (3.4), (3.6) and apply result (3.9) for $f=\mathbb{P} \sigma(u)-\sigma(u)$, we obtain

$$
\|h-u\|_{H^{2}} \leq C\|\mathbb{P} \sigma(u)-\sigma(u)\|_{2} \leq C\|u\|_{H^{1}} \leq C\|u\|_{V} .
$$

Combining the above estimates for $h$ and $h-u$, we deduce (3.10). 
2) Since $\operatorname{curl} \sigma(v) \in L^{2}(\mathcal{O})$ and $\nabla \cdot(\operatorname{curl} \sigma(v))=0$, there exists a unique vector-potential $\psi \in H^{1}(\mathcal{O})$ such that

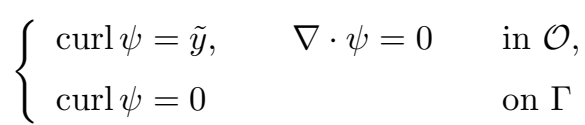

and

$$
\|\psi\|_{H^{1}} \leq C\|\operatorname{curl} \sigma(v)\|_{2}
$$

It follows that $\operatorname{curl}(v-\alpha \Delta v-\psi)=0$ and there exists $\pi \in L^{2}(\mathcal{O})$, such that $v-\alpha \Delta v-$ $\psi+\nabla \pi=0$. Hence $v$ is the solution of the Stokes system

$$
\begin{cases}v-\alpha \Delta v+\nabla \pi=\psi, \quad \operatorname{div} v=0, & \text { in } \mathcal{O} \\ v \cdot \mathrm{n}=0, \quad(\mathrm{n} \cdot D v) \cdot \tau=0 & \text { on } \Gamma .\end{cases}
$$

As a consequence of (3.9), we have

$$
\|v\|_{H^{3}} \leq C\|\psi-v\|_{H^{1}} \leq C\left(\|\psi\|_{H^{1}}+\|v\|_{H^{1}}\right) .
$$

Using (3.14) we state

$$
\|v\|_{H^{3}} \leq C\left(\|\operatorname{curl} \sigma(v)\|_{2}+\|v\|_{H^{1}}\right),
$$

that implies the claimed result (3.11).

3) To prove (3.12) it is enough to show the existence of a positive constant $C$, satisfying the following estimate

$$
\|u\|_{2} \leq C\left(\|\operatorname{curl} \sigma(u)\|_{2}+\|D u\|_{2}\right), \quad \forall u \in \widetilde{W} .
$$

Let us assume that this estimate is not valid, then for any $n \in \mathbb{N}$ there exists a vector-function $v_{n} \in \widetilde{W}$, such that

$$
\left\|v_{n}\right\|_{2} \geq n\left(\left\|\operatorname{curl} \sigma\left(v_{n}\right)\right\|_{2}+\left\|D v_{n}\right\|_{2}\right) .
$$

If we denote $z_{n}=\frac{v_{n}}{\left\|v_{n}\right\|_{2}}$, then the sequence $\left\{z_{n}\right\}_{n=1}^{\infty}$ is uniformly bounded in $\widetilde{W}$

$$
\left\|z_{n}\right\|_{2}=1, \quad\left\|\operatorname{curl} \sigma\left(z_{n}\right)\right\|_{2}+\left\|D z_{n}\right\|_{2} \leq \frac{1}{n} .
$$

Having $\widetilde{W} \subset H^{3}(\mathcal{O})$ and the compact embedding $H^{3}(\mathcal{O}) \hookrightarrow L^{2}(\mathcal{O})$, there exists a subsequence $\left\{z_{n}\right\}_{n=1}^{\infty}$ such that

$$
\begin{array}{lll}
z_{n} \rightarrow z & \text { weakly in } \widetilde{W} \\
z_{n} \rightarrow z & \text { strongly in } L^{2}(\mathcal{O})
\end{array}
$$

From (3.17) we have

$$
\|z\|_{2}=1, \quad\|\operatorname{curl} \sigma(z)\|_{2}+\|D z\|_{2}=0 .
$$

Taking into account that $z \cdot \mathrm{n}=0$ on $\Gamma$, we easily derive that $z=0$, yielding a contradiction with $\|z\|_{2}=1$. Therefore estimate (3.12) holds.

Now, we turn our attention to the nonlinear operators and introduce the operator

$$
b(u, v, \phi)=(u \cdot \nabla v, \phi)
$$

which is well known in the context of Navier-Stokes equations. 
The next two results deal with the properties of the nonlinear operators that will appear in the linearized and backward adjoint equations. Due to these results, we will be able to give a meaning to the stochastic linearized and backward adjoint equations in the variational form, as well as to deduce uniform estimates for the Galerkin approximations (we refer to [2], 3] and [9] for more details).

Lemma 3.5 For any $u, v \in \widetilde{W}$ and $\phi \in V$, we have

$$
(\operatorname{curl} \sigma(v) \times u, \phi)=b(\phi, u, \sigma(v))-b(u, \phi, \sigma(v)) .
$$

Moreover, if $u \in \widetilde{W}$ and $v \in W$ the following estimate holds

$$
|(\operatorname{curl} \sigma(v) \times u, v)| \leq C\|u\|_{H^{3}}\|v\|_{H^{1}}^{2} \leq C_{1}\|u\|_{\widetilde{W}}\|v\|_{V}^{2}
$$

Proof. Taking into account that the vector fields $v$ and $\phi$ have zero divergence and are tangent to the boundary $\Gamma$, identity (3.18) follows by standard computations, integrating by parts the right hand side. In order to derive property (3.19) we set

$$
\begin{aligned}
(\operatorname{curl} \sigma(v) \times u, v) & =b(v, u, \sigma(v))-b(u, v, \sigma(v)) \\
& =b(v, u, v)-b(u, v, v)-\alpha(b(v, u, \Delta v)-b(u, v, \Delta v)) .
\end{aligned}
$$

Integrating by parts and using the boundary conditions, we derive

$$
\begin{aligned}
b(u, v, \Delta v) & =\sum_{i, j=1}^{2} \int_{\mathcal{O}} u_{i} \frac{\partial v_{j}}{\partial x_{i}} \Delta v_{j} d x=\sum_{i, j, k=1}^{2} \int_{\mathcal{O}} u_{i} \frac{\partial v_{j}}{\partial x_{i}} \frac{\partial}{\partial x_{k}}\left(\frac{\partial v_{j}}{\partial x_{k}}-\frac{\partial v_{k}}{\partial x_{j}}\right) d x \\
& =\sum_{i, j, k=1}^{2} \int_{\mathcal{O}} u_{i} \frac{\partial v_{j}}{\partial x_{i}} \frac{\partial}{\partial x_{k}}\left(A_{j k}(v)\right) d x \\
& =\sum_{i, j, k=1}^{2} \int_{\Gamma} u_{i} \frac{\partial v_{j}}{\partial x_{i}} A_{j k}(v) n_{k} d x-\sum_{i, j, k=1}^{2} \int_{\mathcal{O}} \frac{\partial}{\partial x_{k}}\left(u_{i} \frac{\partial v_{j}}{\partial x_{i}}\right) A_{j k}(v) d x \\
& =\sum_{i, j=1}^{2} \int_{\Gamma} u_{i} \frac{\partial v_{j}}{\partial x_{i}} g(v) \tau_{j} d x-\sum_{i, j, k=1}^{2} \int_{\mathcal{O}} \frac{\partial u_{i}}{\partial x_{k}} \frac{\partial v_{j}}{\partial x_{i}} A_{j k}(v) d x-\sum_{i, j, k=1}^{2} \int_{\mathcal{O}} u_{i} \frac{\partial^{2} v_{j}}{\partial x_{x} \partial x_{i}} A_{j k}(v) d x \\
& =I_{1}+I_{2}+I_{3} .
\end{aligned}
$$

Again, integrating by parts, it follows that

$$
\begin{aligned}
I_{1} & =\sum_{i, j=1}^{2} \int_{\Gamma} u_{i} \frac{\partial v_{j}}{\partial x_{i}} g(v) \tau_{j} d x=\sum_{i, j=1}^{2} \int_{\mathcal{O}} u_{i} \frac{\partial v_{j}}{\partial x_{i}} g(v) \tau_{j} \operatorname{div} \mathrm{n} d x+\sum_{i, j, k=1}^{2} \int_{\mathcal{O}} \frac{\partial}{\partial x_{k}}\left(u_{i} \frac{\partial v_{j}}{\partial x_{i}} g(v) \tau_{j}\right) n_{k} d x \\
& =b(u, v, \operatorname{div} \operatorname{ng} g(v) \tau)+\sum_{k=1}^{2} b\left(\frac{\partial u}{\partial x_{k}}, v, n_{k} g(v) \tau\right)-\sum_{k=1}^{2} b\left(u, n_{k} g(v) \tau, \frac{\partial v}{\partial x_{k}}\right) \\
& +\sum_{k=1}^{2} b\left(u, v, n_{k} \frac{\partial}{\partial x_{k}}(g(v) \tau)\right)
\end{aligned}
$$


It is clear that $I_{2}=-\sum_{k=1}^{2} b\left(\frac{\partial u}{\partial x_{k}}, v, A_{k}(v)\right) \quad$ and $\quad I_{3}=0$. Moreover, analogous computations can be performed in order to obtain

$$
\begin{aligned}
b(v, u, \Delta v) & =\sum_{i, j=1}^{2} \int_{\mathcal{O}} v_{i} \frac{\partial u_{j}}{\partial x_{i}} \Delta v_{j} d x=\sum_{i, j, k=1}^{2} \int_{\mathcal{O}} v_{i} \frac{\partial u_{j}}{\partial x_{i}} \frac{\partial}{\partial x_{k}} A_{j k}(v) d x \\
& =\sum_{i, j, k=1}^{2} \int_{\Gamma} v_{i} \frac{\partial u_{j}}{\partial x_{i}} A_{j k}(v) n_{k} d x-\sum_{i, j, k=1}^{2} \int_{\mathcal{O}} \frac{\partial}{\partial x_{k}}\left(v_{i} \frac{\partial u_{j}}{\partial x_{i}}\right) A_{j k}(v) d x \\
& =\sum_{i, j=1}^{2} \int_{\Gamma} v_{i} \frac{\partial u_{j}}{\partial x_{i}} g(v) \tau_{j} d x-\sum_{i, j, k=1}^{2} \int_{\mathcal{O}} \frac{\partial v_{i}}{\partial x_{k}} \frac{\partial u_{j}}{\partial x_{i}} A_{j k}(v)-\sum_{i, j, k=1}^{2} \int_{\mathcal{O}} v_{i} \frac{\partial^{1} u_{j}}{\partial x_{k} \partial x_{i}} A_{j k}(v) d x \\
& =\sum_{i, j=1}^{2} \int_{\Gamma} v_{i} \frac{\partial u_{j}}{\partial x_{i}} g(v) \tau_{j} d x-\sum_{k=1}^{2} b\left(\frac{\partial v}{\partial x_{k}}, u, A_{\cdot k}(v)\right)-\sum_{k=1}^{2} b\left(v, \frac{\partial u}{\partial x_{k}}, A_{\cdot k}(v)\right) .
\end{aligned}
$$

Therefore, from one hand, we have

$$
\begin{aligned}
|b(u, v, \Delta v)| & \leq|b(u, v, \operatorname{div} \operatorname{ng}(v) \tau)|+\sum_{k=1}^{2}\left|b\left(\frac{\partial u}{\partial x_{k}}, v, n_{k} g(v) \tau\right)\right|+\sum_{k=1}^{2}\left|b\left(u, n_{k} g(v) \tau, \frac{\partial v}{\partial x_{k}}\right)\right| \\
& +\sum_{k=1}^{2}\left|b\left(u, v, n_{k} \frac{\partial}{\partial x_{k}}(g(v) \tau)\right)\right|+\sum_{k=1}^{2}\left|b\left(\frac{\partial u}{\partial x_{k}}, v, A \cdot k(v)\right)\right| \\
& \leq C\|u\|_{H^{2}}\|v\|_{H^{1}}^{2} .
\end{aligned}
$$

On other hand, taking into account the trace theorem $\|v\|_{L^{2}(\Gamma)} \leq C\|v\|_{H^{1}}$, we deduce

$$
\begin{aligned}
|b(v, u, \Delta v)| & \leq \sum_{i, j=1}^{2} \int_{\Gamma}\left|v_{i} \frac{\partial u_{j}}{\partial x_{i}} g(v) \tau_{j}\right| d x+\sum_{k=1}^{2}\left(\left|b\left(\frac{\partial v}{\partial x_{k}}, u, A \cdot_{k}(v)\right)\right|+\left|b\left(v, \frac{\partial u}{\partial x_{k}}, A_{k}(v)\right)\right|\right) \\
& \leq 2\|k\|_{C(\overline{\mathcal{O}})}\|\mathrm{n}\|_{C(\overline{\mathcal{O}})}^{2}\|v\|_{L^{2}(\Gamma)}^{2}\|\nabla u\|_{C(\overline{\mathcal{O}})}+\|\nabla v\|_{2}^{2}\|\nabla u\|_{\infty}+\|v\|_{4}\|\nabla u\|_{1,4}\|\nabla v\|_{2} \\
& \leq\left(2 C\|k\|_{C(\overline{\mathcal{O}})}\|\mathrm{n}\|_{C(\overline{\mathcal{O}})}^{2}+1\right)\|\nabla v\|_{2}^{2}\|\nabla u\|_{\infty}+\|v\|_{4}\|\nabla u\|_{1,4}\|\nabla v\|_{2} \\
& \leq C\|u\|_{H^{3}}\|v\|_{H^{1}}^{2} .
\end{aligned}
$$

Then the claimed result is a consequence of (3.6), (3.11) and (3.20)-(3.23).

Also in the following sections we will need the following results.

Lemma 3.6 For any $u, v \in \widetilde{W}$ and $\phi \in V$, we have

$$
\begin{aligned}
(\operatorname{curl}(\sigma(u \times v)), \phi) & =b(\sigma(v), u, \phi)+b(u, \phi, \sigma(v))-b(\sigma(u), v, \phi)+b(v, \sigma(u), \phi)+b(u, v, \phi) \\
& -b(v, u, \phi)-2 \alpha \sum_{i=1}^{2}\left(b\left(\frac{\partial v}{\partial x_{i}}, \frac{\partial u}{\partial x_{i}}, \phi\right)-b\left(\frac{\partial u}{\partial x_{i}}, \frac{\partial v}{\partial x_{i}}, \phi\right)\right) \\
(\operatorname{curl} \sigma(u \times v), \phi) & =b(v, u, \sigma(\phi))-b(u, v, \sigma(\phi))
\end{aligned}
$$

Moreover the following estimate hods

$$
|(\operatorname{curl}(\sigma(u \times v)), \phi)| \leq C\|u\|_{H^{3}}\|v\|_{H^{2}}\|\phi\|_{H^{1}} .
$$


Proof. We verify equality (3.24). Since $\operatorname{curl}(\sigma(u \times v))=\operatorname{curl}(u \times v-\alpha \Delta(u \times v))$, the algebraic equality

$$
\Delta(u \times v)=u \times \Delta v-v \times \Delta u-2 \sum_{i=1}^{2} \frac{\partial v}{\partial x_{i}} \times \frac{\partial u}{\partial x_{i}}
$$

yields

$$
\operatorname{curl}(\sigma(u \times v))=\operatorname{curl}(u \times \sigma(v))-\operatorname{curl}(v \times \sigma(u))+\operatorname{curl}(v \times u)+2 \alpha \sum_{i=1}^{2} \operatorname{curl}\left(\frac{\partial v}{\partial x_{i}} \times \frac{\partial u}{\partial x_{i}}\right) .
$$

Then using the identity

$$
\operatorname{curl}(\varphi \times \psi)=\psi \cdot \nabla \varphi-\varphi \cdot \nabla \psi
$$

for divergence free vector fields $\varphi, \psi$, we deduce

$$
\begin{aligned}
\operatorname{curl}(\sigma(u \times v)) & =\sigma(v) \cdot \nabla u-u \cdot \nabla(\sigma(v))-\sigma(u) \cdot \nabla v+v \cdot \nabla(\sigma(u))+u \cdot \nabla v-v \cdot \nabla u \\
& -2 \alpha \sum_{i=1}^{2}\left(\frac{\partial v}{\partial x_{i}} \cdot \nabla\left(\frac{\partial u}{\partial x_{i}}\right)-\frac{\partial u}{\partial x_{i}} \cdot \nabla\left(\frac{\partial v}{\partial x_{i}}\right)\right),
\end{aligned}
$$

which implies (3.24).

Now, we show (3.25). We assume that $u, v \in W \cap H^{3}(\mathcal{O})$. If $u, v$ are just in $H^{3}(\mathcal{O})$, the result follows from a standard regularization procedure. We have

$$
(\operatorname{curl} \sigma(u \times v), \phi)=(\operatorname{curl}(u \times v), \phi)-\alpha(\operatorname{curl} \Delta(u \times v), \phi) .
$$

Knowing that $\Delta=-$ curl curl, integrating twicely by parts and using relation (3.27), we deduce

$$
\begin{aligned}
(\operatorname{curl}(\Delta(u \times v)), \phi) & =b(v, u, \Delta \phi)-b(u, v, \Delta \phi) \\
& +\int_{\Gamma}(\Delta(u \times v) \times \phi) \cdot \mathrm{n} d x-\int_{\Gamma}(\operatorname{curl}(u \times v) \times \operatorname{curl} \phi) \cdot \mathrm{n} d x .
\end{aligned}
$$

Algebraic calculations show that

$$
\begin{aligned}
\Delta(u \times v) & =u \cdot \nabla(\operatorname{curl} v)-v \cdot \nabla(\operatorname{curl} u)+2 \sum_{k=1}^{2}\left(\nabla u_{k} \times \nabla v_{k}\right), \\
\operatorname{curl}(u \times v) & =v \cdot \nabla u-u \cdot \nabla v .
\end{aligned}
$$

The boundary condition (3.1) on $\Gamma$ implies that

$$
\begin{aligned}
& \Delta(u \times v)=(0,0, u \cdot \nabla g(v)-v \cdot \nabla g(u))+2 \sum_{k=1}^{2}\left(\nabla u_{k} \times \nabla v_{k}\right) \\
& =2(0,0, k(u \cdot \nabla v) \cdot \tau+(u \cdot \nabla(k \tau)) \cdot v-k(v \cdot \nabla u) \cdot \tau-(v \cdot \nabla(k \tau)) \cdot u)+2 \sum_{k=1}^{2}\left(\nabla u_{k} \times \nabla v_{k}\right) .
\end{aligned}
$$

Since $v$ and $u$ are tangent to the boundary $\Gamma$, we obtain

$$
\begin{aligned}
(\Delta(u \times v) \times \phi) \cdot \mathrm{n} & =-2 k(u \cdot \nabla v-v \cdot \nabla u) \cdot \tau(\phi \cdot \tau)+2 \sum_{k=1}^{2}\left(\left(\nabla u_{k} \times \nabla v_{k}\right) \times \phi\right) \cdot \mathrm{n} \\
& -2\{[(u \cdot \tau)(v \cdot \tau)(\tau \cdot \nabla g) \cdot \tau-(v \cdot \tau)(u \cdot \tau)(\tau \cdot \nabla(k \tau)) \cdot \tau](\phi \cdot \tau)\} \\
& =-2 k(u \cdot \nabla v-v \cdot \nabla u) \cdot \tau(\phi \cdot \tau)+2 \sum_{k=1}^{2}\left(\left(\nabla u_{k} \times \nabla v_{k}\right) \times \phi\right) \cdot \mathrm{n} .
\end{aligned}
$$


Using the same reasoning and (3.29) we may verify that on $\Gamma$ the following relation holds

$$
\begin{aligned}
\operatorname{curl}(u \times v) \times \operatorname{curl} \phi \cdot \mathrm{n} & =(v \cdot \nabla u-u \cdot \nabla v) \times(0,0, g(\phi)) \cdot \mathrm{n} \\
& =-2 k(u \cdot \nabla v-v \cdot \nabla u)(\phi \cdot \tau) \cdot \tau .
\end{aligned}
$$

Therefore, the difference of the boundary terms is given by

$$
\int_{\Gamma}(\Delta(u \times v) \times \phi) \cdot \mathrm{n} d x-\int_{\Gamma}(\operatorname{curl}(u \times v) \times \operatorname{curl} \phi) \cdot \mathrm{n} d x=2 \sum_{k=1}^{2} \int_{\Gamma}\left(\nabla u_{k} \times \nabla v_{k}\right) \times \phi \cdot \mathrm{n} d x .
$$

On the other hand, the boundary condition $(n \cdot D v) \cdot \tau=0$ and $\operatorname{div} v=0$ imply

$$
\frac{\partial v_{1}}{\partial x_{2}}+\frac{\partial v_{2}}{\partial x_{1}}=\frac{4 n_{1} n_{2}}{n_{2}^{2}-n_{1}^{2}} \frac{\partial v_{1}}{\partial x_{1}} \quad \text { on } \Gamma,
$$

which gives

$$
\sum_{k=1}^{2}\left(\nabla u_{k} \times \nabla v_{k}\right) \times\left.\phi \cdot \mathrm{n}\right|_{\Gamma}=\left(\frac{\partial u_{1}}{\partial x_{1}}\left(\frac{\partial v_{1}}{\partial x_{2}}+\frac{\partial v_{2}}{\partial x_{1}}\right)-\frac{\partial v_{1}}{\partial x_{1}}\left(\frac{\partial u_{1}}{\partial x_{2}}+\frac{\partial u_{2}}{\partial x_{1}}\right)\right) \phi \cdot \tau=0 .
$$

Therefore the difference of the boundary terms in (3.28) vanishes. As a consequence of it we derive

$$
(\operatorname{curl} \sigma(u \times v), \phi)=(\operatorname{curl}(u \times v), \phi)-\alpha(b(v, u, \Delta \phi)-b(u, v, \Delta \phi)) .
$$

Then using (3.29), we deduce (3.25).

Finally, estimate (3.26) results from (3.25), by taking into account the anti-symmetry of the operator $b$ with respect to the second and third variables, and the Sobolev embedding results.

\section{Well-posedness of the state equation}

The stochastic differential equation (2.1) has been studied in [9. In this section, we recall the notion of the solution, as well as the properties of the solution that will be relevant in the study of the control problem.

Assume that the stochastic noise is represented by

$$
G(t, y) d W_{t}=\sum_{k=1}^{m} g^{k}(t, y) d W_{t}^{k},
$$

where

$$
\begin{aligned}
G(t, y) & =\left(g^{1}(\cdot, y), \ldots, g^{m}(\cdot, y)\right):[0, T] \times V \rightarrow V^{m}=\overbrace{V \times \ldots \times V}^{m-\text { times }}, \\
g^{k}(\cdot, y) & \in L^{2}(0, T ; V) \quad \text { for any } y \in V .
\end{aligned}
$$

Let us define the norm $\|G(t, y)\|_{V}=\sum_{i=1}^{m}\left\|g^{i}(t, y)\right\|_{V}$. We also assume that $G(t, y)$ is Gâteaux differentiable in the variable $y$

$$
\lim _{s \rightarrow 0} \frac{G(t, y+s v)-G(t, y)}{s}=\nabla_{y} G(t, y) v \quad \text { for each } t \in[0, T],
$$


and, moreover, for each $t \in[0, T]$ the function $\nabla_{y} G(t, y)$ is continuous and bounded in the second variable $y \in V$, namely

$$
\left\|\nabla_{y} G(t, x)-\nabla_{y} G(t, y)\right\|_{V} \rightarrow 0 \quad \text { when } \quad\|x-y\|_{V} \rightarrow 0
$$

and

$$
\left\|\nabla_{y} G(t, y) v\right\|_{V} \leq C\|v\|_{V}, \quad v \in V
$$

for some positive constant $C$.

Due to Propositions A.2, A.3 of [1] we have that $G:[0, T] \times V \rightarrow V^{m}$ is Lipschitz and Fréchet differentiable in the second variable $y \in V$, that is there exists a positive constant $K$ such that

$$
\begin{aligned}
\|G(t, y)-G(t, z)\|_{V} & \leq K\|y-z\|_{V}, \\
G(t, x+y)-G(t, x)-\nabla_{y} G(t, x) y & =O\left(t,\|y\|_{V}\right), \quad \forall x, y \in V, \quad t \in[0, T],
\end{aligned}
$$

having the property

$$
\frac{O(t, s)}{s} \rightarrow 0 \quad \text { in } V, \quad \text { as } \quad s \rightarrow 0 .
$$

Definition 4.1 Let $U \in L^{2}\left(\Omega, L^{2}\left(0, T ; H^{1}(\mathcal{O})\right)\right)$ and $Y_{0} \in L^{2}(\Omega, \widetilde{W})$. A stochastic process $Y \in L^{2}\left(\Omega, L^{\infty}(0, T ; \widetilde{W})\right)$ is a strong solution of (2.1), if for a.e.-P and a.e. $t \in(0, T)$, the following equation holds

$$
\begin{aligned}
(\sigma(Y(t)), \phi)= & \int_{0}^{t}[-2 \nu(D Y(s), D \phi)-(\operatorname{curl} \sigma(Y(s)) \times Y(s), \phi)] d s \\
& +(\sigma(Y(0)), \phi)+\int_{0}^{t}(U(s), \phi) d s+\int_{0}^{t}(G(s, Y(s)), \phi) d W_{s}
\end{aligned}
$$

for all $\phi \in V$, where the nonlinear term is understood in the following sense

$$
\begin{aligned}
(\operatorname{curl} \sigma(Y(t)) \times Y(t), \phi) & =b(\phi, Y(t), Y(t)-\alpha \Delta Y(t))-b(Y(t), \phi, Y(t)-\alpha \Delta Y(t)) \\
& =b(Y(t), Y(t), \phi)-\alpha(b(\phi, Y(t), \Delta Y(t))-b(Y(t), \phi, \Delta Y(t))) .
\end{aligned}
$$

The following theorem has been proved in the article [9].

Theorem 4.2 Assume that

$$
U \in L^{p}\left(\Omega, L^{p}\left(0, T ; H^{1}(\mathcal{O})\right)\right) \quad \text { and } \quad Y_{0} \in L^{p}(\Omega, V) \cap L^{2}(\Omega, \widetilde{W}) \quad \text { for some } 4 \leq p<\infty .
$$

Then there exists a unique strong solution $Y$ to system (2.1), which belongs to

$$
L^{2}\left(\Omega, L^{\infty}(0, T ; \widetilde{W})\right) \cap L^{p}\left(\Omega, L^{\infty}(0, T ; V)\right) .
$$

Moreover, the following estimates hold

$$
\begin{gathered}
\mathbb{E} \sup _{s \in[0, t]}\|Y(s)\|_{V}^{2}+8 \nu \mathbb{E} \int_{0}^{t}\|D Y(s)\|_{2}^{2} d s \leq C\left(\mathbb{E}\left\|Y_{0}\right\|_{V}^{2}+\mathbb{E}\|U\|_{L^{2}\left(0, t ; L^{2}\right)}^{2}+1\right), \\
\mathbb{E} \sup _{s \in[0, t]}\|\operatorname{curl} \sigma(Y(s))\|_{2}^{2} \leq C\left(\mathbb{E}\left\|\operatorname{curl} \sigma\left(Y_{0}\right)\right\|_{2}^{2}+\mathbb{E}\|U\|_{L^{2}\left(0, t ; H^{1}\right)}^{2}+1\right), \\
\mathbb{E} \sup _{s \in[0, t]}\|Y(s)\|_{V}^{p} \leq C \mathbb{E}\left\|Y_{0}\right\|_{V}^{p}+C\left(1+\mathbb{E} \int_{0}^{t}\|U(s)\|_{2}^{p} d s\right) .
\end{gathered}
$$


In Sections 5,8 we consider that the data $U, Y_{0}$ satisfy assumptions (4.4) and denote the unique solution of (4.3) by

$$
Y \in L^{p}\left(\Omega, L^{\infty}(0, T ; V)\right) \cap L^{2}\left(\Omega, L^{\infty}(0, T ; \widetilde{W})\right)
$$

which satisfies estimates (4.5).

We also recall the stability result of solutions for the stochastic differential system (2.1) obtained in [9].

Proposition 4.3 Assume that

$$
U_{1}, U_{2} \in L^{p}\left(\Omega, L^{p}\left(0, T ; H^{1}(\mathcal{O})\right)\right) \quad \text { for } 4 \leq p<\infty .
$$

Let

$$
Y_{1}, Y_{2} \in L^{2}\left(\Omega, L^{\infty}(0, T ; \widetilde{W})\right)
$$

be the corresponding solutions of (2.1) with the same initial condition

$$
Y_{0} \in L^{p}(\Omega, V) \cap L^{2}(\Omega, \widetilde{W}) .
$$

Then there exist positive constants $C$ and $C_{0}$, such that the following estimate holds for a.e. $t \in(0, T)$

$$
\mathbb{E} \sup _{s \in[0, t]} \xi_{0}(s)\left\|Y_{1}(s)-Y_{2}(s)\right\|_{W}^{2} \leq C \mathbb{E} \int_{0}^{t} \xi_{0}(s)\left\|U_{1}(s)-U_{2}(s)\right\|_{2}^{2} d s,
$$

with the function $\xi_{0}$ defined as

$$
\xi_{0}(t)=e^{-C_{0} \int_{0}^{t}\left(\left\|Y_{1}\right\|_{H^{3}}+\left\|Y_{2}\right\|_{H^{3}}+1\right) d s} .
$$

\section{Stochastic linearized state equation}

In the study of the control problem the well-posedness of the stochastic linearized state equation is a crucial step, since such a solution will correspond to the Gâteaux derivative of the control to state map. However the mathematical analysis of this equation is not an easy issue.

Let us take

$$
\Psi \in L^{2}(\Omega \times(0, T) \times \mathcal{O})
$$

and consider the following linear system

$$
\begin{cases}d \sigma(Z)=(\nu \Delta Z-\operatorname{curl} \sigma(Z) \times Y-\operatorname{curl} \sigma(Y) \times Z-\nabla \pi+\Psi) d t & \\ \operatorname{div} Z=0 & +\nabla_{y} G(t, Y) Z d W_{t}, \\ Z \cdot \mathrm{n}=0, \quad(\mathrm{n} \cdot D Z) \cdot \tau=0 & \text { in }(0, T) \times \mathcal{O}, \\ Z(0)=0 & \text { in }(0, T) \times \Gamma,\end{cases}
$$

The difficulties are related with the regularity of the coefficients in equation (5.2) $)_{1}$. Firstly we should give a correct meaning to the solution. Next, taking an appropriate basis on the functional space $H^{2}$ we follow the Galerkin method to construct the approximate solution. Deriving uniform estimates for the Galerkin approximations we will be able to pass to the limit and obtain the solvability for the stochastic linearized state system (5.2). 
Definition 5.1 A stochastic process $Z \in L^{2}(0, T ; W)$ is a strong solution of (5.2) if for $P$-a.e. $\omega \in \Omega$, a.e. $t \in(0, T)$ the following equality holds

$$
\begin{aligned}
(\sigma(Z(t)), \phi) & =\int_{0}^{t}\{-2 \nu(D Z(s), D \phi) \\
& -b(\phi, Y(s), \sigma(Z(s)))+b(Y(s), \phi, \sigma(Z(s))) \\
& -b(\phi, Z(s), \sigma(Y(s)))+b(Z(s), \phi, \sigma(Y(s)))+(\Psi(s), \phi)\} d s \\
& +\int_{0}^{t}\left(\nabla_{y} G(s, Y(s)) Z(s), \phi\right) d W_{s} \quad \text { for any } \phi \in V .
\end{aligned}
$$

Let us recall that the proof of Theorem 4.2 in $[9$ has been given by the Galerkin approximation method taking a basis $\left\{e_{i}\right\} \subset \widetilde{W}$ of eigenfunctions to the injection operator $I: \widetilde{W} \hookrightarrow V$. Here, to construct the solution for the stochastic differential equation (5.2) we follow analogous strategy. We define an appropriate basis which is different of the previous one.

Since the injection operator $I: W \hookrightarrow V$ is a compact operator, there exists a basis $\left\{h_{i}\right\} \subset W$ of eigenfunctions

$$
\left(v, h_{i}\right)_{W}=\mu_{i}\left(v, h_{i}\right)_{V}, \quad \forall v \in W, \quad i \in \mathbb{N},
$$

being simultaneously an orthonormal basis for $V$. The corresponding sequence $\left\{\mu_{i}\right\}$ of eigenvalues fulfills the properties: $\mu_{i}>0, \forall i \in \mathbb{N}$, and $\mu_{i} \rightarrow \infty$ as $i \rightarrow \infty$. We may consider $\left\{h_{i}\right\} \subset H^{4}$, taking into account that the ellipticity of equation (5.4) increases the regularity of their solutions (see [6]).

Let us consider the basis $\left\{h_{i}\right\}$ and introduce the Galerkin approximations of equation (5.2). Let $W_{n}=\operatorname{span}\left\{h_{1}, \ldots, h_{n}\right\}$ and define

$$
Z_{n}(t)=\sum_{i=1}^{n} \zeta_{i}(t) h_{i} \quad \text { for each } t \in[0, T],
$$

as the solution of the stochastic differential equation

$$
\begin{gathered}
d \sigma\left(Z_{n}\right)=\left(\nu \Delta Z_{n}-\operatorname{curl} \sigma\left(Z_{n}\right) \times\right. \\
+\nabla_{y} G(t, Y) Z_{n} d W_{t} .
\end{gathered}
$$

This means that

$$
\begin{aligned}
d\left(\sigma\left(Z_{n}\right), \phi\right) & =\left(\left(\nu \Delta Z_{n}-\operatorname{curl} \sigma\left(Z_{n}\right) \times Y-\operatorname{curl} \sigma(Y) \times Z_{n}+\Psi\right), \phi\right) d t \\
& +\left(\nabla_{y} G(t, Y) Z_{n}, \phi\right) d W_{t} \quad \text { for each } \phi \in W_{n} .
\end{aligned}
$$

Equation (5.5) defines a system of stochastic linear ordinary differential equations, which has a unique solution $Z_{n}$ as an adapted process in the space $C\left([0, T] ; W_{n}\right)$.

Let us show the following result.

Proposition 5.2 Assume that $\Psi$ satisfies assumption (5.1). Then equation (5.5) admits a unique solution $Z_{n}$. Moreover, there exist positive constants $C_{1}, C_{2}$ and $C$, which are independent on the index $n$, such that the following estimates hold for each $t \in[0, T]$

$$
\begin{aligned}
\mathbb{E} \sup _{s \in[0, t]} \xi_{1}(s)\left\|Z_{n}(s)\right\|_{V}^{2}+ & \mathbb{E} \int_{0}^{t} \xi_{1}(s)\left\|D Z_{n}\right\|^{2} d s \leq C \mathbb{E} \int_{0}^{t} \xi_{1}(s)\|\Psi\|_{2}^{2} d s, \\
\mathbb{E} \sup _{s \in[0, t]} \xi_{2}(s)\left\|Z_{n}(s)\right\|_{W}^{2} & \leq C \mathbb{E} \int_{0}^{t} \xi_{2}(s)\|\Psi\|_{2}^{2} d s,
\end{aligned}
$$


where the functions $\xi_{1}, \xi_{2}$ are defined as

$$
\xi_{1}(t)=e^{-2 C_{1} \int_{0}^{t}\|Y\|_{\widetilde{W}} d s}, \quad \xi_{2}(t)=e^{-4 C_{2} \int_{0}^{t}\|Y\|_{\widetilde{W}} d s} .
$$

Proof. 1st step: Estimate in the space $V$ for $Z_{n}$.

In order to simplify the notation, let us introduce the function

$$
f\left(Z_{n}\right)=\nu \Delta Z_{n}-\operatorname{curl} \sigma\left(Z_{n}\right) \times Y-\operatorname{curl} \sigma(Y) \times Z_{n}+\Psi,
$$

which belongs to $H^{1}$. Setting $\phi=h_{i}$ in equation (5.5), we obtain

$$
d\left(Z_{n}, h_{i}\right)_{V}=\left(f\left(Z_{n}\right), h_{i}\right) d t+\left(\nabla_{y} G(t, Y) Z_{n}, h_{i}\right) d W_{t} .
$$

By Itô's formula we have

$$
\begin{aligned}
d\left(Z_{n}, h_{i}\right)_{V}^{2}=2\left(Z_{n}, h_{i}\right)_{V}\left(f\left(Z_{n}\right), h_{i}\right) d t & +\left(\nabla_{y} G(t, Y) Z_{n}, h_{i}\right)^{2} d t \\
+ & 2\left(Z_{n}, h_{i}\right)_{V}\left(\nabla_{y} G(t, Y) Z_{n}, h_{i}\right) d W_{t} .
\end{aligned}
$$

Summing these equalities on $i=1, \ldots, n$ and using the property

$$
2\left(f\left(Z_{n}\right), Z_{n}\right)=-4 \nu\left\|D Z_{n}\right\|_{2}^{2}-2\left(\operatorname{curl} \sigma\left(Z_{n}\right) \times Y-\Psi, Z_{n}\right),
$$

we derive

$$
\begin{aligned}
d\left\|Z_{n}\right\|_{V}^{2} & =\left(-4 \nu\left\|D Z_{n}\right\|_{2}^{2}-2\left(\operatorname{curl} \sigma\left(Z_{n}\right) \times Y-\Psi, Z_{n}\right)\right) d t \\
& +\sum_{i=1}^{n}\left(\nabla_{y} G(t, Y) Z_{n}, h_{i}\right)^{2} d t+2\left(\nabla_{y} G(t, Y) Z_{n}, Z_{n}\right) d W_{t} .
\end{aligned}
$$

By (3.19) we have

$$
\left|\left(\operatorname{curl} \sigma\left(Z_{n}\right) \times Y, Z_{n}\right)\right| \leq C_{1}\|Y\|_{\widetilde{W}}\left\|Z_{n}\right\|_{V}^{2},
$$

therefore multiplying (5.8) by

$$
\xi_{1}(t)=e^{-2 C_{1} \int_{0}^{t}\|Y\|_{\widetilde{W}} d s}
$$

and using Itô's formula, we obtain

$$
\begin{aligned}
d\left(\xi_{1}(t)\left\|Z_{n}\right\|_{V}^{2}\right) & =-2 C_{1} \xi_{1}(t)\|Y\|_{\widetilde{W}}\left\|Z_{n}\right\|_{V}^{2} d t \\
& -\xi_{1}(t)\left(4 \nu\left\|D Z_{n}\right\|_{2}^{2}+2\left(\operatorname{curl} \sigma\left(Z_{n}\right) \times Y-\Psi, Z_{n}\right)\right) d t \\
& +\xi_{1}(t) \sum_{i=1}^{n}\left(\nabla_{y} G(t, Y) Z_{n}, h_{i}\right)^{2} d t \\
& +2 \xi_{1}(t)\left(\nabla_{y} G(t, Y) Z_{n}, Z_{n}\right) d W_{t} .
\end{aligned}
$$

Estimate (5.9) and the integration over the time variable imply

$$
\begin{aligned}
\xi_{1}(t)\left\|Z_{n}(t)\right\|_{V}^{2} & +4 \nu \int_{0}^{t} \xi_{1}\left\|D Z_{n}\right\|_{2}^{2} d s \leq 2 \int_{0}^{t} \xi_{1}\left|\left(\Psi, Z_{n}\right)\right| d s \\
& +\int_{0}^{t} \xi_{1} \sum_{i=1}^{n}\left(\nabla_{y} G(s, Y) Z_{n}, h_{i}\right)^{2} d s \\
& +2\left|\int_{0}^{t} \xi_{1}\left(\nabla_{y} G(s, Y) Z_{n}, Z_{n}\right) d W_{s}\right| .
\end{aligned}
$$


Let

$$
\tau_{N}(\omega)=\inf \left\{t \in[0, T]:\left\|Z_{n}(t)\right\|_{W} \geq N\right\}
$$

be the stopping time for fixed $N \in \mathbb{N}$. The Burkholder-Davis-Gundy inequality and property (4.1) give

$$
\begin{aligned}
\mathbb{E} \sup _{s \in\left[0, \tau_{N} \wedge t\right]} \mid & \left|\int_{0}^{s} \xi_{1}(r)\left(\nabla_{y} G(r, Y) Z_{n}, Z_{n}\right) d W_{r}\right| \\
& \leq \mathbb{E}\left(\int_{0}^{\tau_{N} \wedge t} \xi_{1}^{2}(s)\left(\nabla_{y} G(s, Y) Z_{n}, Z_{n}\right)^{2} d s\right)^{\frac{1}{2}} \\
& \leq C \mathbb{E} \sup _{s \in\left[0, \tau_{N} \wedge t\right]} \sqrt{\xi_{1}(s)}\left\|Z_{n}\right\|_{2}\left(\int_{0}^{\tau_{N} \wedge t} \xi_{1}(s)\left\|Z_{n}\right\|_{V}^{2} d s\right)^{\frac{1}{2}} \\
& \leq \varepsilon \mathbb{E} \sup _{s \in\left[0, \tau_{N} \wedge t\right]} \xi_{1}(s)\left\|Z_{n}\right\|_{V}^{2}+C_{\varepsilon} \mathbb{E} \int_{0}^{\tau_{N} \wedge t} \xi_{1}(s)\left\|Z_{n}\right\|_{V}^{2} d s .
\end{aligned}
$$

Substituting this inequality with $\varepsilon=\frac{1}{2}$ in (5.10), taking the supremum on $s \in\left[0, \tau_{N} \wedge t\right]$ and the expectation, we deduce

$$
\begin{aligned}
\frac{1}{2} \mathbb{E} \sup _{s \in\left[0, \tau_{N} \wedge t\right]} \xi_{1}(s)\left\|Z_{n}(s)\right\|_{V}^{2} & +4 \nu \mathbb{E} \int_{0}^{\tau_{N} \wedge t} \xi_{1}\left\|D Z_{n}\right\|_{2}^{2} d s \\
& \leq C \mathbb{E} \int_{0}^{\tau_{N} \wedge t} \xi_{1}\left\|Z_{n}\right\|_{V}^{2} d s+C \mathbb{E} \int_{0}^{t} \xi_{1}\|\Psi\|_{2}^{2} d s .
\end{aligned}
$$

Hence the function

$$
f(t)=\mathbb{E} \sup _{s \in\left[0, \tau_{N} \wedge t\right]} \xi_{1}(s)\left\|Z_{n}(s)\right\|_{V}^{2}
$$

fulfills Gronwall's type inequality

$$
\frac{1}{2} f(t) \leq C \int_{0}^{t} f(s) d s+C \mathbb{E} \int_{0}^{t} \xi_{1}(s)\|\Psi\|_{2}^{2} d s .
$$

The application of Gronwall's inequality gives

$$
\begin{aligned}
\mathbb{E} \sup _{s \in\left[0, \tau_{N} \wedge t\right]} \xi_{1}(s)\left\|Z_{n}(s)\right\|_{V}^{2} & +8 \nu \mathbb{E} \int_{0}^{\tau_{N} \wedge t} \xi_{1}\left\|D Z_{n}\right\|_{2}^{2} d s \\
& \leq C \mathbb{E} \int_{0}^{t} \xi_{1}\|\Psi\|_{2}^{2} d s .
\end{aligned}
$$

2nd step: Estimate in the space $W$ for $Z_{n}$.

Let $\tilde{f}_{n}$ and $\widetilde{G}_{n}$ be the solutions of problem (3.8) for $f=f\left(Z_{n}\right)$ and $f=\nabla_{y} G(t, Y) Z_{n}$, respectively. The following relations hold

$$
\left(\tilde{f}_{n}, h_{i}\right)_{V}=\left(f\left(Z_{n}\right), h_{i}\right), \quad\left(\widetilde{G}_{n}, h_{i}\right)_{V}=\left(\nabla_{y} G(t, Y) Z_{n}, h_{i}\right) \quad \text { for each } i .
$$

Using relations (5.4) and multiplying (5.7) by $\mu_{i}$, we deduce

$$
d\left(Z_{n}, h_{i}\right)_{W}=\left(\tilde{f}_{n}, h_{i}\right)_{W} d t+\left(\widetilde{G}_{n}, h_{i}\right)_{W} d W_{t} .
$$


Hence the Itô formula gives

$$
\begin{aligned}
d\left(Z_{n}, h_{i}\right)_{W}^{2} & =2\left(Z_{n}, h_{i}\right)_{W}\left(\tilde{f}_{n}, h_{i}\right)_{W} d t+\left(\widetilde{G}_{n}, h_{i}\right)_{W}^{2} d t \\
& +2\left(Z_{n}, h_{i}\right)_{W}\left(\widetilde{G}_{n}, h_{i}\right)_{W} d W_{t} .
\end{aligned}
$$

Multiplying these equalities by $\frac{1}{\mu_{i}}$ and summing over $i=1, \ldots, n$, we obtain

$$
d\left\|Z_{n}\right\|_{W}^{2}=2\left(\tilde{f}_{n}, Z_{n}\right)_{W} d t+\left\|\widetilde{G}_{n}\right\|_{W}^{2} d t+2\left(\widetilde{G}_{n}, Z_{n}\right)_{W} d W_{t} .
$$

Then, by the relation between the norms of the spaces $W$ and $V$, induced by the inner products (2.3), we derive

$$
\begin{aligned}
d\left(\left\|Z_{n}\right\|_{W}^{2}\right)=2\left(\left(\mathbb{P} \sigma\left(\tilde{f}_{n}\right), \mathbb{P} \sigma\left(Z_{n}\right)\right)\right. & \left.+\left(\tilde{f}_{n}, Z_{n}\right)_{V}\right) d t+\left\|\widetilde{G}_{n}\right\|_{W}^{2} d t \\
& +2\left(\left(\mathbb{P} \sigma\left(\widetilde{G}_{n}\right), \mathbb{P} \sigma\left(Z_{n}\right)\right)+\left(\widetilde{G}_{n}, Z_{n}\right)_{V}\right) d W_{t} .
\end{aligned}
$$

Using (5.12), this equality can be written as

$$
\begin{aligned}
d\left(\left\|Z_{n}\right\|_{W}^{2}\right) & =2\left(\left(f\left(Z_{n}\right), \mathbb{P} \sigma\left(Z_{n}\right)\right)+\left(f\left(Z_{n}\right), Z_{n}\right)\right) d t+\left\|\widetilde{G}_{n}\right\|_{W}^{2} d t \\
& +2\left[\left(\nabla_{y} G(t, Y) Z_{n}, \mathbb{P} \sigma\left(Z_{n}\right)\right)+\left(\nabla_{y} G(t, Y) Z_{n}, Z_{n}\right)\right] d W_{t} .
\end{aligned}
$$

First let us estimate the most difficult term $\left(\operatorname{curl} \sigma\left(Z_{n}\right) \times Y, \mathbb{P} \sigma\left(Z_{n}\right)\right)$, being a part of the expression $\left(f\left(Z_{n}\right), \mathbb{P} \sigma\left(Z_{n}\right)\right)$. If we denote $R=\sigma\left(Z_{n}\right)$ and $Q=\mathbb{P} \sigma\left(Z_{n}\right)-\sigma\left(Z_{n}\right)$, applying (3.18) we get

$$
\begin{aligned}
\left|\left(\operatorname{curl} \sigma\left(Z_{n}\right) \times Y, \mathbb{P} \sigma\left(Z_{n}\right)\right)\right| & \leq|(\operatorname{curl} R \times Y, Q)|+|(\operatorname{curl} R \times Y, R)| \\
& \leq|b(Q, Y, R)-b(Y, Q, R)|+|b(R, Y, R)-b(Y, R, R)| \\
& \leq|b(Q, Y, R)|+|b(Y, Q, R)|+|b(R, Y, R)| \\
& \leq C\|Y\|_{1, \infty}\left(\|Q\|_{H^{1}}\|R\|_{2}+\|R\|_{2}^{2}\right) .
\end{aligned}
$$

Hence

$$
\left|\left(\operatorname{curl} \sigma\left(Z_{n}\right) \times Y, \mathbb{P} \sigma\left(Z_{n}\right)\right)\right| \leq C\|Y\|_{1, \infty}\left\|Z_{n}\right\|_{W}^{2} \leq \widetilde{C}_{2}\|Y\|_{\widetilde{W}}\left\|Z_{n}\right\|_{W}^{2},
$$

by using estimates (3.4)-(3.5) for $v=Z_{n}$. Obviously we have

$$
\left|\left(\operatorname{curl} \sigma(Y) \times Z_{n}, \mathbb{P} \sigma\left(Z_{n}\right)\right)\right| \leq C\|Y\|_{H^{3}}\left\|Z_{n}\right\|_{\infty}\left\|\mathbb{P} \sigma\left(Z_{n}\right)\right\|_{W}^{2} \leq \bar{C}_{2}\|Y\|_{\widetilde{W}}\left\|Z_{n}\right\|_{W}^{2} .
$$

Recalling that $f\left(Z_{n}\right)=\nu \Delta Z_{n}-\operatorname{curl} \sigma\left(Z_{n}\right) \times Y-\operatorname{curl} \sigma(Y) \times Z_{n}+\Psi$ and (5.9), we deduce

$$
\begin{aligned}
\left|\left(f\left(Z_{n}\right), \mathbb{P} \sigma\left(Z_{n}\right)\right)\right| & \leq C\left\|Z_{n}\right\|_{W}^{2}+C_{2}\|Y\|_{\widetilde{W}}\left\|Z_{n}\right\|_{W}^{2}+\left|\left(\Psi, \mathbb{P} \sigma\left(Z_{n}\right)\right)\right|, \\
\left|\left(f\left(Z_{n}\right), Z_{n}\right)\right| & \leq-2 \nu\left\|D Z_{n}\right\|_{2}^{2}+C_{1}\|Y\|_{\widetilde{W}}\left\|Z_{n}\right\|_{V}^{2}+\left|\left(\Psi, Z_{n}\right)\right| \\
& \leq C_{2}\|Y\|_{\widetilde{W}}\left\|Z_{n}\right\|_{W}^{2}+\left|\left(\Psi, Z_{n}\right)\right|
\end{aligned}
$$

for

$$
C_{2}=\max \left(\widetilde{C}_{2}, \bar{C}_{2}, C_{1}\right)
$$


As in previous considerations, if we take the function $\xi_{2}(t)=e^{-4 C_{2} \int_{0}^{t}\|Y\|_{\widetilde{W}} d s}$ and use Ito's formula, then (5.13) and (5.14) imply

$$
\begin{aligned}
d\left(\xi_{2}(t)\left\|Z_{n}\right\|_{W}^{2}\right) & \leq C \xi_{2}(t)\left(\left\|Z_{n}\right\|_{W}^{2}+\left|\left(\Psi, \mathbb{P} \sigma\left(Z_{n}\right)\right)\right|+\left|\left(\Psi, Z_{n}\right)\right|\right) d t \\
& +\xi_{2}(t)\left\|\widetilde{G}_{n}\right\|_{W}^{2} d t \\
& \left.+2 \xi_{2}(t)\left(\nabla_{y} G(t, Y) Z_{n}, \mathbb{P} \sigma\left(Z_{n}\right)+Z_{n}\right)\right) d W_{t}
\end{aligned}
$$

Therefore the integration of this inequality over the time variable gives

$$
\begin{aligned}
\xi_{2}(t)\left\|Z_{n}(t)\right\|_{W}^{2} \leq & C \int_{0}^{t} \xi_{2}\left(\left\|Z_{n}\right\|_{W}^{2}+\|\Psi\|_{2}^{2}\right) d s+\int_{0}^{t} \xi_{2}\left\|\widetilde{G}_{n}\right\|_{W}^{2} d s \\
& +2\left|\int_{0}^{t} \xi_{2}\left(\nabla_{y} G(s, Y) Z_{n}, \mathbb{P} \sigma\left(Z_{n}\right)+Z_{n}\right) d W_{s}\right| .
\end{aligned}
$$

Since $\widetilde{G}_{n}$ is the solution of the Stokes type equation (3.8), then using (4.1) we have

$$
\left\|\widetilde{G}_{n}\right\|_{W} \leq C\left\|Z_{n}\right\|_{V}
$$

Moreover the Burkholder-Davis-Gundy inequality gives

$$
\begin{aligned}
\mathbb{E} \sup _{s \in\left[0, \tau_{N} \wedge t\right]}\left|\int_{0}^{s} \xi_{2}(r)\left(\nabla_{y} G(r, Y) Z_{n}, \mathbb{P} \sigma\left(Z_{n}\right)+Z_{n}\right) d W_{r}\right| \\
\leq \mathbb{E}\left(\int_{0}^{\tau_{N} \wedge t} \xi_{2}^{2}(s)\left(\nabla_{y} G(s, Y) Z_{n}, \mathbb{P} \sigma\left(Z_{n}\right)+Z_{n}\right)^{2} d s\right)^{\frac{1}{2}} \\
\leq C \mathbb{E} \sup _{s \in\left[0, \tau_{N} \wedge t\right]} \sqrt{\xi_{2}(s)}\left\|Z_{n}\right\|_{W}\left(\int_{0}^{\tau_{N} \wedge t} \xi_{2}(s)\left\|Z_{n}\right\|_{2}^{2} d s\right)^{\frac{1}{2}} \\
\leq \varepsilon \mathbb{E} \sup _{s \in\left[0, \tau_{N} \wedge t\right]} \xi_{2}(s)\left\|Z_{n}\right\|_{W}^{2}+C_{\varepsilon} \mathbb{E} \int_{0}^{\tau_{N} \wedge t} \xi_{2}(s)\left\|Z_{n}\right\|_{W}^{2} d s .
\end{aligned}
$$

Substituting inequalities (5.16) and (5.17) with chosen $\varepsilon=\frac{1}{2}$ in (5.15) and taking the supremum on $s \in\left[0, \tau_{N} \wedge t\right]$, we derive

$$
\frac{1}{2} \mathbb{E} \sup _{s \in\left[0, \tau_{N} \wedge t\right]} \xi_{2}(s)\left\|Z_{n}(s)\right\|_{W}^{2} \leq C \mathbb{E} \int_{0}^{\tau_{N} \wedge t} \xi_{2}(s)\left\|Z_{n}\right\|_{W}^{2} d s+C \mathbb{E} \int_{0}^{t} \xi_{2}(s)\|\Psi\|_{2}^{2} d s .
$$

The application of Gronwall's inequality gives

$$
\mathbb{E} \sup _{s \in\left[0, \tau_{N} \wedge t\right]} \xi_{2}(s)\left\|Z_{n}(s)\right\|_{W}^{2} \leq C \mathbb{E} \int_{0}^{t} \xi_{2}\|\Psi\|_{2}^{2} d s .
$$

Hence passing to the limit $N \rightarrow \infty$ in (5.11) and (5.18), we derive estimates (5.6).

As a direct consequence of Proposition 5.2 we derive the following existence result for the stochastic linearized state equation (5.2).

Theorem 5.3 Assume that $\Psi$ satisfies assumption (5.1). Then there exists a unique solution $Z$ to system (5.2), such that

$$
Z \in L^{\infty}(0, T ; W) \text { for } \quad P \text {-a.e. } \omega \in \Omega,
$$


satisfying the following a priori estimates

$$
\begin{gathered}
\mathbb{E} \sup _{s \in[0, t]} \xi_{1}(s)\|Z(s)\|_{V}^{2}+\mathbb{E} \int_{0}^{t} \xi_{1}\|Z\|_{V}^{2} d s \leq C \mathbb{E} \int_{0}^{t} \xi_{1}\|\Psi\|_{2}^{2} d s \quad \text { for a.e. } t \in(0, T), \\
\mathbb{E} \sup _{s \in[0, T]} \xi_{2}(s)\|Z(s)\|_{W}^{2} \leq C \mathbb{E} \int_{0}^{T} \xi_{2}\|\Psi\|_{2}^{2} d s .
\end{gathered}
$$

Proof. Since

$$
\sup _{t \in[0, T]}\|Y(t)\|_{\widetilde{W}}^{2} \leq C(\omega) \quad \text { for all } \omega \in \Omega \backslash A, \quad \text { where } P(A)=0
$$

by (2.3) and (4.5), therefore there exists a positive constant $K(\omega)$, which depends only on $\omega \in$ $\Omega \backslash A$ and satisfies

$$
0<K(\omega) \leq \xi_{i}(t) \leq 1 \quad \text { for all } \omega \in \Omega \backslash A, \quad t \in[0, T], \quad i=1,2 .
$$

By a priori estimates of Lemma 5.2 we have

$$
\mathbb{E} \sup _{t \in[0, T]} \xi_{1}(t)\left\|Z_{n}(t)\right\|_{V}^{2} \leq C, \quad \mathbb{E} \sup _{t \in[0, T]} \xi_{2}(t)\left\|Z_{n}(t)\right\|_{W}^{2} \leq C .
$$

Hence using (5.19) there exists a suitable subsequence $Z_{n}$, indexed by the same index $n$, such that

$$
\begin{aligned}
\sqrt{\xi_{1}(t)} Z_{n} & \rightarrow \sqrt{\xi_{1}(t)} Z \quad{ }_{\text {-weakly in } L^{2}\left(\Omega, L^{\infty}(0, T ; V)\right),} \\
S_{n}=\sqrt{\xi_{2}(t)} Z_{n} & \rightarrow S=\sqrt{\xi_{2}(t)} Z \quad \text { *-weakly in } L^{2}\left(\Omega, L^{\infty}(0, T ; W)\right) .
\end{aligned}
$$

Since $Z_{n}$ solves (5.5), then we have

$$
\begin{aligned}
d\left(\sigma\left(Z_{n}\right), \phi\right) & =\left(\left(\nu \Delta Z_{n}-\operatorname{curl} \sigma\left(Z_{n}\right) \times Y-\operatorname{curl} \sigma(Y) \times Z_{n}+\Psi\right), \phi\right) d t \\
& +\left(\nabla_{y} G(t, Y) Z_{n}, \phi\right) d W_{t} \quad \text { for each } \quad \phi \in W_{n} .
\end{aligned}
$$

Using Ito's formula for $\xi(t)=\sqrt{\xi_{2}(t)}=e^{-2 C_{2} \int_{0}^{t}\|Y\|_{\widetilde{W}} d s}$, we get

$$
\begin{aligned}
d\left(\sigma\left(S_{n}\right), \phi\right) & =\left[\left(\left\{\nu \Delta S_{n}-\operatorname{curl} \sigma\left(S_{n}\right) \times Y-\operatorname{curl} \sigma(Y) \times S_{n}+\xi \Psi\right\}, \phi\right)\right. \\
& \left.-2 C_{2}\|Y\|_{\widetilde{W}} \quad\left(\sigma\left(S_{n}\right), \phi\right)\right] d t+\left(\nabla_{y} G(t, Y) S_{n}, \phi\right) d W_{t},
\end{aligned}
$$

that is

$$
\begin{aligned}
\left(\sigma\left(S_{n}(t)\right), \phi\right) & =\int_{0}^{t}\left[-2 C_{2}\|Y\|_{\widetilde{W}}\left(\sigma\left(S_{n}\right), \phi\right)-2 \nu\left(D S_{n}, D \phi\right)\right. \\
& -b\left(\phi, Y, \sigma\left(S_{n}\right)\right)+b\left(Y, \phi, \sigma\left(S_{n}\right)\right) \\
& \left.-b\left(\phi, S_{n}, \sigma(Y)\right)+b\left(S_{n}, \phi, \sigma(Y)\right)+(\xi \Psi, \phi)\right] d s \\
& +\int_{0}^{t}\left(\nabla_{y} G(s, Y) S_{n}, \phi\right) d W_{s} .
\end{aligned}
$$


Let us consider an arbitrary $\varphi=\varphi(\omega) \in L^{2}(\Omega)$. Multiplying this equality by $\varphi$ and taking the expectation, we derive

$$
\begin{aligned}
\mathbb{E} \varphi(\omega)\left(\sigma\left(S_{n}(t)\right), \phi\right) & =\mathbb{E} \varphi(\omega)\left\{\int _ { 0 } ^ { t } \left[-2 C_{2}\|Y\|_{\widetilde{W}}\left(\sigma\left(S_{n}\right), \phi\right)-2 \nu\left(D S_{n}, D \phi\right)\right.\right. \\
& -b\left(\phi, Y, \sigma\left(S_{n}\right)\right)+b\left(Y, \phi, \sigma\left(S_{n}\right)\right) \\
& \left.-b\left(\phi, S_{n}, \sigma(Y)\right)+b\left(S_{n}, \phi, \sigma(Y)\right)+(\xi \Psi, \phi)\right] d s \\
& \left.+\int_{0}^{t}\left(\nabla_{y} G(s, Y) S_{n}(s), \phi\right) d W_{s}\right\} \quad \text { for } \forall t \in[0, T] .
\end{aligned}
$$

Using that the right side of the last equation is continuous in the time variable $t \in[0, T]$ and applying (5.20) and Proposition A.3, p.93 of [4], we pass to the limit $n \rightarrow \infty$ in this equality and deduce

$$
\begin{aligned}
\mathbb{E} \varphi(\omega)(\sigma(S(t)), \phi) & =\mathbb{E} \varphi(\omega)\left\{\int _ { 0 } ^ { t } \left[-2 C_{2}\|Y\|_{\widetilde{W}}(\sigma(S), \phi)-2 \nu(D S, D \phi)\right.\right. \\
& -b(\phi, Y, \sigma(S))+b(Y, \phi, \sigma(S)) \\
& -b(\phi, S, \sigma(Y))+b(S, \phi, \sigma(Y))+(\xi \Psi, \phi)] d s \\
& \left.+\int_{0}^{t}\left(\nabla_{y} G(s, Y) S, \phi\right) d W_{s}\right\} .
\end{aligned}
$$

Since $\varphi \in L^{2}(\Omega)$ is arbitrary and $S(t)=\xi(t) Z(t)$, then we have the validity of the equality

$$
\begin{aligned}
\xi(t)(\sigma(Z(t)), \phi) & =\left\{\int _ { 0 } ^ { t } \xi \left[-2 C_{2}\|Y\|_{\widetilde{W}}(\sigma(S), \phi) d s-2 \nu(D Z, D \phi)\right.\right. \\
& -b(\phi, Y, \sigma(Z))+b(Y, \phi, \sigma(Z)) \\
& -b(\phi, Z, \sigma(Y))+b(Z, \phi, \sigma(Y))+(\Psi, \phi)] d s \\
& \left.+\int_{0}^{t} \xi(s)\left(\nabla_{y} G(s, Y) Z, \phi\right) d W_{s}\right\} \quad \text { for } \forall t \in[0, T] \quad \text { and } P \text {-a.e. } \quad \omega \in \Omega .
\end{aligned}
$$

Hence

$$
\begin{aligned}
d[\xi(\sigma(Z), \phi)] & =\xi\left[-2 C_{2}\|Y\|_{\widetilde{W}}(\sigma(Z), \phi)-2 \nu(D Z, D \phi)\right. \\
& -b(\phi, Y, \sigma(Z))+b(Y, \phi, \sigma(Z)) \\
& -b(\phi, Z, \sigma(Y))+b(Z, \phi, \sigma(Y))+(\Psi, \phi)] d t \\
& \left.+\left(\nabla_{y} G(s, Y) Z, \phi\right) d W_{s}\right\} \quad \text { for a.e. } t \in[0, T] \quad \text { and } \quad P \text {-a.e. } \quad \omega \in \Omega .
\end{aligned}
$$

Moreover if we use Ito's formula

$$
d(\sigma(Z), \phi)=d[\widehat{\xi} \xi(\sigma(Z), \phi)]=\xi(\sigma(Z), \phi) d \widehat{\xi}+\widehat{\xi} d[\xi(\sigma(Z), \phi)]
$$

for $\widehat{\xi}(t)=e^{2 C_{2} \int_{0}^{t}\|Y\|_{\widetilde{W}} d s}$, we derive

$$
\begin{aligned}
d(\sigma(Z), \phi) & =\{[-2 \nu(D Z, D \phi) \\
& -b(\phi, Y, \sigma(Z))+b(Y, \phi, \sigma(Z)) \\
& -b(\phi, Z, \sigma(Y))+b(Z, \phi, \sigma(Y))+(\Psi, \phi)] d t \\
& \left.+\left(\nabla_{y} G(t, Y) Z, \phi\right) d W_{t}\right\} \quad \text { for a.e. } t \in[0, T], P \text {-a.e. } \quad \omega \in \Omega
\end{aligned}
$$

and for each $\phi \in W$. Therefore the stochastic process $Z \in L^{2}(0, T ; W)$ is a solution of (5.2) in the sense of equality (5.3). 


\section{Gâteaux differentiability of the control-to-state mapping}

It is well known that the Gâteaux derivative of the control-to-state is fundamental to deduce the necessary optimality conditions.

The goal of this section is to prove that the Gâteaux derivative of $Y$ at the point $U$, in the direction $\Psi$, is defined as the solution of the linear system (5.2). We know that due to Theorem 5.3 this system has a unique solution. As a consequence of this theorem and Proposition 4.3 we have the following result.

Proposition 6.1 Given $U, Y_{0}$, satisfying (4.4) and

$$
\Psi \in L^{p}\left(\Omega, L^{p}\left(0, T ; H^{1}(\mathcal{O})\right)\right) \quad \text { for } 4 \leq p<\infty,
$$

let us consider

$$
U_{\rho}=U+\rho \Psi, \quad \forall \rho \in(0,1) .
$$

If $Y$ and $Y_{\rho}$ are the solutions of (2.1) corresponding to $\left(U, Y_{0}\right)$ and $\left(U_{\rho}, Y_{0}\right)$, then the following representation holds

$$
Y_{\rho}=Y+\rho Z+\rho \delta_{\rho} \quad \text { with } \quad \lim _{\rho \rightarrow 0} \sup _{t \in[0, T]}\left\|\delta_{\rho}\right\|_{V}^{2}=0 \quad P \text {-a.e. } \quad \omega \in \Omega,
$$

where

$$
Z \in L^{\infty}(0, T ; W) \text { for } \quad P \text {-a.e. } \omega \in \Omega,
$$

is the solution of (5.2), satisfying the estimates of Theorem 5.3.

Proof. It is straightforward to verify that $Z_{\rho}=\frac{Y_{\rho}-Y}{\rho}$ satisfies the equation

$$
\begin{aligned}
d \sigma\left(Z_{\rho}\right) & =\left(\nu \Delta Z_{\rho}-\operatorname{curl} \sigma\left(Z_{\rho}\right) \times Y-\operatorname{curl} \sigma\left(Y_{\rho}\right) \times Z_{\rho}-\nabla \pi_{\rho}+\Psi\right) d t \\
& +\frac{1}{\rho}\left(G\left(t, Y_{\rho}\right)-G(t, Y)\right) d W_{t} .
\end{aligned}
$$

If we consider the solution $Z$ of (5.2), then $\delta_{\rho}=Z_{\rho}-Z$ is the solution of the equation

$$
\begin{aligned}
d \sigma\left(\delta_{\rho}\right) & =\left(\nu \Delta \delta_{\rho}-\operatorname{curl} \sigma\left(\delta_{\rho}\right) \times Y-\operatorname{curl} \sigma\left(Y_{\rho}\right) \times \delta_{\rho}-\operatorname{curl} \sigma\left(Y_{\rho}-Y\right) \times Z-\nabla\left(\pi_{\rho}-\pi\right)\right) d t \\
& +R d W_{t},
\end{aligned}
$$

where

$$
R=\left[\frac{1}{\rho}\left(G\left(t, Y_{\rho}\right)-G(t, Y)\right)-\nabla_{y} G(t, Y) Z\right]
$$

We apply the operator $(I-\alpha \mathbb{P} \Delta)^{-1}$ to equation (6.22) and deduce a stochastic differential equation for $\delta_{\rho}$, then the Itô formula gives

$$
\begin{aligned}
d\left(\left\|\delta_{\rho}\right\|_{V}\right)+2 \nu\left\|D \delta_{\rho}\right\|_{2}^{2} & =-2\left(\left(\operatorname{curl} \sigma\left(\delta_{\rho}\right) \times Y, \delta_{\rho}\right)+\left(\operatorname{curl} \sigma\left(Y_{\rho}\right) \times \delta_{\rho}, \delta_{\rho}\right)\right) d t \\
& -2\left(\operatorname{curl} \sigma\left(Y_{\rho}-Y\right) \times Z, \delta_{\rho}\right) d t+2\left(R, \delta_{\rho}\right) d W_{t} \\
& +\left\|\widetilde{G}_{\rho}-\widetilde{G}\right\|_{V} d t
\end{aligned}
$$

where $\widetilde{G}_{\rho}$ and $\widetilde{G}$ are the solutions of the modified Stokes problem (3.8) with $f$ replaced by $\frac{1}{\rho}\left(G(t, Y)-G\left(t, Y_{\rho}\right)\right)$ and $\nabla_{y} G(t, Y) Z$, respectively. 
Applying (4.2) we have

$R=\nabla_{y} G(t, Y) \delta_{\rho}+o\left(t,\left\|Z+\delta_{\rho}\right\|_{V}\right) \quad$ with $o\left(t,\left\|Z+\delta_{\rho}\right\|_{V}\right) \rightarrow 0 \quad$ in $V, \quad$ as $\quad \rho \rightarrow 0$ for $t \in[0, T]$ and obtain

$$
\left\|\widetilde{G}_{\rho}-\widetilde{G}\right\|_{V}^{2} \leq\|R\|_{2}^{2} \leq C\left\|\delta_{\rho}\right\|_{V}^{2}+o\left(t,\left\|Z+\delta_{\rho}\right\|_{V}^{2}\right) .
$$

Using property (3.18), we have

$$
\left(\operatorname{curl} \sigma\left(Y_{\rho}\right) \times \delta_{\rho}, \delta_{\rho}\right)=b\left(\delta_{\rho}, \delta_{\rho}, \sigma\left(Y_{\rho}\right)\right)-b\left(\delta_{\rho}, \delta_{\rho}, \sigma\left(Y_{\rho}\right)\right)=0
$$

and applying estimate (3.19), we get

$$
\left|\left(\operatorname{curl} \sigma\left(\delta_{\rho}\right) \times Y, \delta_{\rho}\right)\right| \leq C\|Y\|_{\widetilde{W}}\left\|\delta_{\rho}\right\|_{V}^{2} .
$$

In addition, using the Sobolev embedding results, we estimate the term

$$
\begin{aligned}
\left|\left(\operatorname{curl} \sigma\left(Y_{\rho}-Y\right) \times Z, \delta_{\rho}\right)\right| & =\left|b\left(\delta_{\rho}, Z, \sigma\left(Y_{\rho}-Y\right)\right)-b\left(Z, \delta_{\rho}, \sigma\left(Y_{\rho}-Y\right)\right)\right| \\
& \leq\left(\left\|\delta_{\rho}\right\|_{4}\|\nabla Z\|_{4}+\|Z\|_{\infty}\left\|\nabla \delta_{\rho}\right\|_{2}\right)\left\|\sigma\left(Y_{\rho}-Y\right)\right\|_{2} \\
& \leq C\|Z\|_{H^{2}}\left\|\delta_{\rho}\right\|_{V}\left\|\sigma\left(Y_{\rho}-Y\right)\right\|_{2} \\
& \leq C\|Z\|_{W}^{2}\left\|\delta_{\rho}\right\|_{V}^{2}+C\left\|\sigma\left(Y_{\rho}-Y\right)\right\|_{2}^{2} .
\end{aligned}
$$

Introducing the above deduced relations in (6.24), we obtain

$$
\begin{aligned}
d\left(\left\|\delta_{\rho}\right\|_{V}^{2}\right) & \leq C_{4}\left(\|Y\|_{\widetilde{W}}^{2}+\|Z\|_{W}^{2}+1\right)\left\|\delta_{\rho}\right\|_{V}^{2}+C\left\|\sigma\left(Y_{\rho}-Y\right)\right\|_{2}^{2} d t \\
& +\left(R, \delta_{\rho}\right) d W_{t} .
\end{aligned}
$$

Considering the function

$$
\beta(t)=e^{-\widetilde{C}_{4} \int_{0}^{t}\left(\|Y\|_{\widetilde{W}}^{2}+\left\|Y_{\rho}\right\|_{\widetilde{W}}^{2}+\|Z\|_{W}^{2}+1\right) d s} \quad \text { for } \widetilde{C}_{4}=\max \left\{C_{0}, C_{4}\right\},
$$

the Itô formula yields

$$
\beta(t)\left\|\delta_{\rho}(t)\right\|_{V}^{2} \leq C \int_{0}^{t} \beta(s)\left\|\sigma\left(Y_{\rho}-Y\right)\right\|_{2}^{2} d s+\int_{0}^{t} \beta(s)\left(R, \delta_{\rho}\right) d W_{s} .
$$

Moreover the Burkholder-Davis-Gundy inequality, the Young inequality and (6.23) give

$$
\begin{aligned}
\mathbb{E} \sup _{s \in[0, t]} & \left|\int_{0}^{s} \beta(r)\left(R, \delta_{\rho}\right) d W_{r}\right| \leq \mathbb{E}\left(\int_{0}^{t} \beta(r)^{2}\left(R, \delta_{\rho}\right)^{2} d s\right)^{1 / 2} \\
\leq & \varepsilon \mathbb{E} \sup _{s \in[0, t]} \beta(s)\left\|\delta_{\rho}\right\|_{V}^{2}+C_{\varepsilon} \mathbb{E} \int_{0}^{t} \beta(s)\left[\left\|\delta_{\rho}\right\|_{V}^{2}+o\left(s,\left\|Z+\delta_{\rho}\right\|_{V}^{2}\right)\right] d s .
\end{aligned}
$$

Substituting this inequality with $\varepsilon=\frac{1}{2}$ in (6.25), taking the supremum on the time interval $[0, t]$ and the expectation, we obtain

$$
\begin{aligned}
\frac{1}{2} \mathbb{E} \sup _{s \in[0, t]} \beta(s)\left\|\delta_{\rho}(s)\right\|_{V}^{2} \leq & C \mathbb{E} \int_{0}^{t} \beta(s)\left\|\delta_{\rho}\right\|_{V}^{2} d s+C \int_{0}^{t} \beta(s)\left\|\sigma\left(Y_{\rho}-Y\right)\right\|_{2}^{2} d s \\
& +C \mathbb{E} \int_{0}^{t} \beta(s) o\left(s, \rho\left\|Z+\delta_{\rho}\right\|_{V}^{2}\right) d s .
\end{aligned}
$$


Applying Gronwall's inequality, we deduce

$$
\begin{aligned}
\mathbb{E} \sup _{s \in[0, t]} \beta(s)\left\|\delta_{\rho}\right\|_{V}^{2} & \leq C \mathbb{E} \int_{0}^{t} \beta(s)\left\|\sigma\left(Y_{\rho}-Y\right)\right\|_{2}^{2} d s+C \mathbb{E} \int_{0}^{t} \beta(s) o\left(s, \rho\left\|Z+\delta_{\rho}\right\|_{V}^{2}\right) d s \\
& \leq C T \mathbb{E} \sup _{s \in[0, t]} \xi_{0}(s)\left\|\sigma\left(Y_{\rho}-Y\right)\right\|_{2}^{2}+C \mathbb{E} \int_{0}^{t} \beta(s) o\left(s, \rho\left\|Z+\delta_{\rho}\right\|_{V}^{2}\right) d s,
\end{aligned}
$$

where

$$
\xi_{0}(t)=e^{-C_{0} \int_{0}^{t}\left(\left\|Y_{\rho}\right\|_{\widetilde{W}}^{2}+\|Y\|_{\widetilde{W}}^{2}\right) d s}
$$

and $C_{0}$ is defined in Proposition 4.3. The result of Proposition 4.3 is valid for $Y_{1}=Y_{\rho}, Y_{2}=Y$. Therefore we obtain

$$
\begin{aligned}
\mathbb{E} \sup _{s \in[0, t]} \beta(s)\left\|\delta_{\rho}\right\|_{V}^{2} & \leq C T \mathbb{E} \sup _{s \in[0, t]} \xi_{0}(s)\left\|\sigma\left(Y_{\rho}-Y\right)\right\|_{2}^{2} d s \\
& \leq \rho C \mathbb{E} \int_{0}^{t} \xi_{0}\|\Psi\|_{2}^{2} d s+C \mathbb{E} \int_{0}^{t} o\left(s, \rho\left\|Z+\delta_{\rho}\right\|_{V}^{2}\right) d s .
\end{aligned}
$$

Since the right hand side in this inequality converges to zero as $\rho \rightarrow 0$, then applying the Lebesgue theorem, we deduce 6.21).

Let us assume that the Lagrangian $L:[0, T] \times V \times \widetilde{W} \rightarrow \mathbb{R}^{+}$and the function $h: \widetilde{W} \rightarrow \mathbb{R}^{+}$, involved in the cost functional (2.4), satisfy the following hypotheses:

H1) $L(t, u, y)$ and $h(y)$ are Gâteaux differentiable on $y$ for any fixed $u \in V, \quad t \in[0, T]$;

H2) there exist positive constants $C$, such that

$$
\begin{aligned}
\left\|\nabla_{u} L(t, u, y)\right\|_{2}+\left\|\nabla_{y} L(t, u, y)\right\|_{2} & \leq C\left(1+\|u\|_{V}+\|y\|_{\widetilde{W}}\right), \\
\left\|\nabla_{y} h(t, y)\right\|_{2} & \leq C\left(1+\|y\|_{\widetilde{W}}\right), \quad \forall u \in V, y \in \widetilde{W}, t \in[0, T] ;
\end{aligned}
$$

H3) the $L^{1}$-integrability on the time for $L$ and $h$

$$
L(\cdot, u, y), h(y) \in L^{1}(0, T), \quad \forall u \in V, y \in \widetilde{W} .
$$

As a direct consequence of Proposition 6.1 and hypotheses H1)-H3) we easily derive the following result on the variation for the cost functional (2.4).

Proposition 6.2 Let $U, Y_{0}, \Psi$ and $U_{\rho}=U+\rho \Psi$ satisfy the conditions of Proposition 6.1, Let the cost functional (2.4) fulfill hypotheses H1)-H3), then

$$
\begin{aligned}
J\left(U_{\rho}, Y_{\rho}\right)= & J(U, Y)+\rho \mathbb{E} \int_{0}^{T}\left\{\left(\nabla_{u} L(t, U, Y), \Psi\right)+\left(\nabla_{y} L(t, U, Y), Z\right)\right\} d t \\
& +\mathbb{E}\left(\nabla h\left(Y_{T}\right), Z\right)_{V}+o(\rho),
\end{aligned}
$$

where $Y, Y_{\rho}$ are the solutions of (2.1), corresponding to $\left(U, Y_{0}\right),\left(U_{\rho}, Y_{0}\right)$ and $Z$ is the solution of (5.2).

Remark 6.3 As a consequence of (6.26) we have that $L(t, \cdot, \cdot)$ and $h$ are Lipschitz continuous

$$
\begin{aligned}
\left|L\left(t, u_{1}, y_{1}\right)-L\left(t, u_{2}, y_{2}\right)\right| & \leq C\left(1+\max _{i=1,2}\left\|u_{i}\right\|_{V}+\max _{i=1,2}\left\|y_{i}\right\|_{\widetilde{W}}\right)\left(\left\|u_{1}-u_{2}\right\|_{V}+\left\|y_{1}-y_{2}\right\|_{\widetilde{W}}\right), \\
\left|h\left(y_{1}\right)-h\left(y_{2}\right)\right| & \leq C\left(1+\max _{i=1,2}\left\|y_{i}\right\|_{\widetilde{W}}\right)\left(\left\|y_{1}-y_{2}\right\|_{\widetilde{W}}\right), \quad \forall u_{i} \in V, y_{i} \in \widetilde{W}, t \in[0, T] .
\end{aligned}
$$




\section{Stochastic backward adjoint equation}

The aim of this section is to prove the existence of the adjoint stochastic process $(p, q)$, which is related to the Gâteaux derivative of the control to state mapping through a duality condition. Let us consider the following backward stochastic system

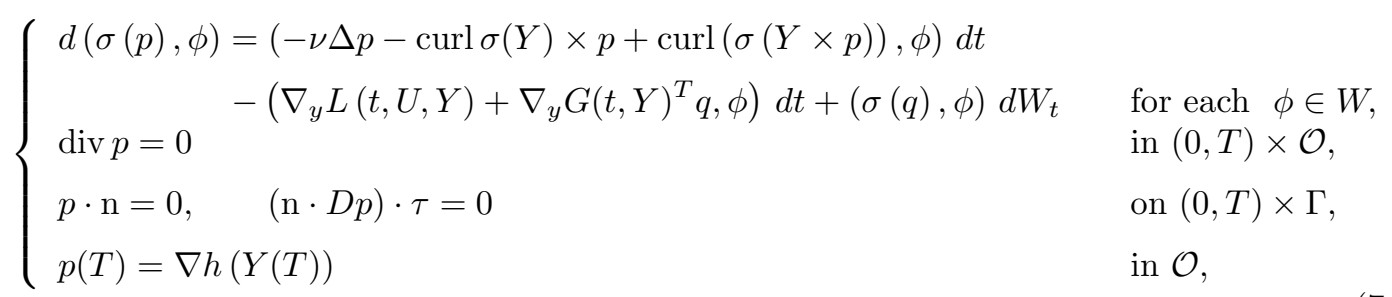

where $Y$ is the unique solution of (4.3).

In all this section we assume that the cost functional (2.4) fulfill hypotheses H1)-H3).

\subsection{Solvability of the adjoint equation}

The solution of (7.1) is understood in the following sense.

Definition 7.1 A stochastic process

$$
(p, q) \in L^{\infty}(0, T ; W) \times L^{2}(0, T ; W) \quad P-\text { a.e. in } \Omega
$$

is a solution of (7.1) if for $P$-a.e. $\omega \in \Omega$ and a.e. $t \in(0, T)$ the following equality holds

$$
\begin{aligned}
(\sigma(\nabla h(Y(T))), \phi)-(\sigma(p(t)), \phi) & =\int_{t}^{T}\{2 \nu(D p(s), D \phi) \\
& -b(\phi, p(s), \sigma(Y(s)))+b(p(s), \phi, \sigma(Y(s))) \\
& +b(p(s), Y(s), \sigma(\phi(s)))-b(Y(s), p(s), \sigma(\phi(s))) \\
& \left.+\left(\nabla_{y} L(t, U, Y)+\nabla_{y} G(t, Y)^{T} q, \phi\right)\right\} d s \\
& +\int_{t}^{T}(\sigma(q), \phi) d W_{s} \quad \text { for each } \quad \phi \in W .
\end{aligned}
$$

We construct the solution of system (7.1), using Galerkin approximations. Let us consider the basis $\left\{h_{i}\right\}$, defined in (5.4), and consider the space $W_{n}=\operatorname{span}\left\{h_{1}, \ldots, h_{n}\right\}$. Let

$$
p_{n}=\sum_{i=1}^{n} \mathfrak{p}_{i}(t) h_{i} \quad \text { and } \quad q_{n}=\sum_{i=1}^{n} \mathfrak{q}_{i}(t) h_{i}
$$

be the solution of the Galerkin approximations of system (7.1), being the following backward 
stochastic system

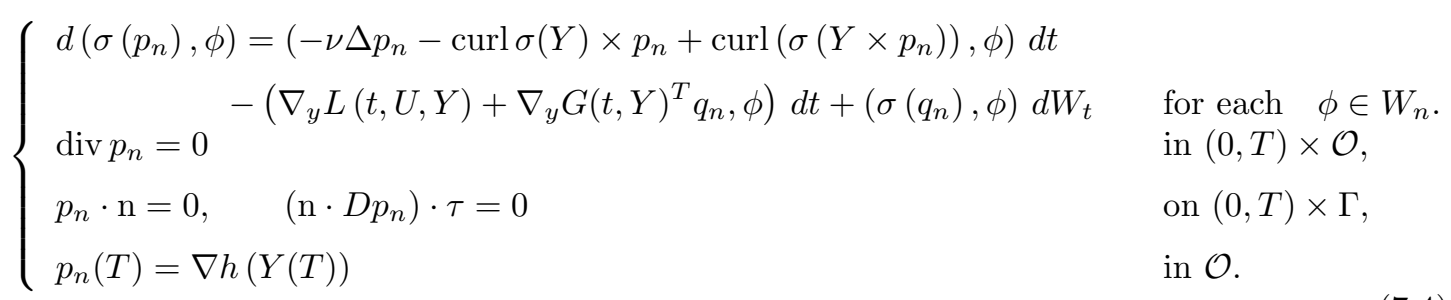

In the next proposition we show the existence of the pair $\left(p_{n}, q_{n}\right)$.

Proposition 7.2 There exists a unique solution

$$
\left(p_{n}, q_{n}\right) \in L^{\infty}(0, T ; W) \times L^{2}(0, T ; W) \quad P-\text { a.e. in } \Omega
$$

of the backward stochastic system (7.4). Moreover there exists a positive constant $C_{3}$, such that the pair $\left(p_{n}, q_{n}\right)$ satisfies the following estimate for a.e. $t \in(0, T)$

$$
\begin{aligned}
\frac{1}{2} \mathbb{E} \sup _{s \in[t, T]} \xi_{3}(s)\left\|p_{n}(s)\right\|_{W}^{2} & +\mathbb{E} \int_{t}^{T} \xi_{3}(s)\left(\frac{\nu}{\alpha}\left\|\mathbb{P} \sigma\left(p_{n}\right)\right\|_{2}^{2}+4 \nu\left\|D p_{n}\right\|_{2}^{2}+\frac{1}{2}\left\|q_{n}\right\|_{W}^{2}\right) d s \\
& \leq C\left(\mathbb{E}\|\nabla h(Y(T))\|_{W}^{2}+\mathbb{E} \int_{t}^{T} \xi_{3}(s)\left\|\nabla_{y} L(s, U, Y)\right\|_{2}^{2} d s\right)(7.5)
\end{aligned}
$$

with the function

$$
\xi_{3}(t)=e^{-C_{3} \int_{t}^{T}\|Y\|_{\widetilde{W}} d s}
$$

Proof. 1st step: Existence of approximate solutions $\left(p_{n}, q_{n}\right)$. Equation (7.4) defines a system of stochastic backward linear ordinary differential equations, which has a unique solution $\left(p_{n}, q_{n}\right)$ as an adapted process in the space $C\left([0, T] ; W_{n}\right)$.

2nd step: Estimate in the space $W$ for the approximate solutions $\left(p_{n}, q_{n}\right)$. Setting $\phi=h_{i}$ in equation (7.4) 1 , we obtain

$$
d\left(p_{n}, h_{i}\right)_{V}=\left(f\left(p_{n}\right), h_{i}\right) d t-\left(\nabla_{y} G(t, Y)^{T} q_{n}, h_{i}\right) d t+\left(q_{n}, h_{i}\right)_{V} d W_{t}
$$

with

$$
f\left(p_{n}\right)=-\nu \Delta p_{n}-\operatorname{curl} \sigma(Y) \times p_{n}+\operatorname{curl}\left(\sigma\left(Y \times p_{n}\right)\right)-\nabla_{y} L(t, U, Y) .
$$

Let $\tilde{f}_{n}, \widetilde{G}_{n}$ be the solutions of the modified Stokes problem (3.8) for $f=f\left(p_{n}\right), f=$ $\nabla_{y} G(t, Y)^{T} q_{n}$, respectively. The following relations hold

$$
\left(\tilde{f}_{n}, h_{i}\right)_{V}=\left(f\left(p_{n}\right), h_{i}\right), \quad\left(\widetilde{G}_{n}, h_{i}\right)_{V}=\left(\nabla_{y} G(t, Y)^{T} q_{n}, h_{i}\right) \quad \text { for each } i .
$$

Using relations (5.4) and multiplying (17.6) by $\mu_{i}$, we deduce

$$
d\left(p_{n}, h_{i}\right)_{W}=\left(\tilde{f}_{n}, h_{i}\right)_{W} d t-\left(\widetilde{G}_{n}, h_{i}\right)_{W} d t+\left(q_{n}, h_{i}\right)_{W} d W_{t} .
$$

On the other hand, the Itô formula gives

$$
\begin{aligned}
d\left(p_{n}, h_{i}\right)_{W}^{2} & =2\left(p_{n}, h_{i}\right)_{W}\left(\tilde{f}_{n}, h_{i}\right)_{W} d t-2\left(p_{n}, h_{i}\right)_{W}\left(\widetilde{G}_{n}, h_{i}\right)_{W} d t \\
& +2\left(p_{n}, h_{i}\right)_{W}\left(q_{n}, h_{i}\right)_{W} d W_{t}+\left(q_{n}, h_{i}\right)_{W}^{2} d t .
\end{aligned}
$$


Multiplying these equalities by $\frac{1}{\mu_{i}}$ and summing over $i=1, \ldots, n$, we obtain

$$
d\left\|p_{n}\right\|_{W}^{2}=2\left(\tilde{f}_{n}, p_{n}\right)_{W} d t-2\left(\widetilde{G}_{n}, p_{n}\right)_{W} d t+2\left(q_{n}, p_{n}\right)_{W} d W_{t}+\left\|q_{n}\right\|_{W}^{2} d t .
$$

Then, by the relation between the inner products for the spaces $W$ and $V$, we derive

$$
\begin{aligned}
d\left\|p_{n}\right\|_{W}^{2}=2\left(\left(\mathbb{P} \sigma\left(\tilde{f}_{n}\right), \mathbb{P} \sigma\left(p_{n}\right)\right)\right. & \left.+\left(\tilde{f}_{n}, p_{n}\right)_{V}\right) d t-2\left[\left(\mathbb{P} \sigma\left(\widetilde{G}_{n}\right), \mathbb{P} \sigma\left(p_{n}\right)\right)+\left(\widetilde{G}_{n}, p_{n}\right)_{V}\right] d t \\
& +2\left(\left(\mathbb{P} \sigma\left(q_{n}\right), \mathbb{P} \sigma\left(p_{n}\right)\right)+\left(q_{n}, p_{n}\right)_{V}\right) d W_{t}+\left\|q_{n}\right\|_{W}^{2} d t
\end{aligned}
$$

Using (17.8), this equality can be written as

$$
\begin{aligned}
d\left\|p_{n}\right\|_{W}^{2} & =2\left[\left(f\left(p_{n}\right), \mathbb{P} \sigma\left(p_{n}\right)\right)+\left(f\left(p_{n}\right), p_{n}\right)\right] d t-2\left[\left(\nabla_{y} G(t, Y)^{T} q_{n}, \mathbb{P} \sigma\left(p_{n}\right)\right)\right. \\
& \left.\left.+\left(\nabla_{y} G(t, Y)^{T} q_{n}, p_{n}\right)\right] d t+2\left(\sigma\left(q_{n}\right), \mathbb{P} \sigma\left(p_{n}\right)+p_{n}\right)\right) d W_{t}+\left\|q_{n}\right\|_{W}^{2} d t .
\end{aligned}
$$

In what follows we estimate the terms of the right hand side in this equality. From (7.7) we have that

$$
\left(f\left(p_{n}\right), p_{n}\right)=2 \nu\left\|D p_{n}\right\|_{2}^{2}+\left(\operatorname{curl}\left(\sigma\left(Y \times p_{n}\right)\right), p_{n}\right)-\left(\nabla_{y} L(t, U, Y), p_{n}\right)
$$

and

$$
\begin{aligned}
\left(f\left(p_{n}\right), \mathbb{P} \sigma\left(p_{n}\right)\right) & =\frac{\nu}{\alpha}\left\|\mathbb{P} \sigma\left(p_{n}\right)\right\|_{2}^{2}-\frac{\nu}{\alpha}\left(p_{n}, \mathbb{P} \sigma\left(p_{n}\right)\right)-\left(\operatorname{curl} \sigma(Y) \times p_{n}, \mathbb{P} \sigma\left(p_{n}\right)\right) \\
& +\left(\operatorname{curl}\left(\sigma\left(Y \times p_{n}\right)\right), \mathbb{P} \sigma\left(p_{n}\right)\right)-\left(\nabla_{y} L(t, U, Y), \mathbb{P} \sigma\left(p_{n}\right)\right) .
\end{aligned}
$$

Since

$$
\begin{aligned}
\left|\left(\operatorname{curl} \sigma(Y) \times p_{n}, \mathbb{P} \sigma\left(p_{n}\right)\right)\right| & \leq\|\operatorname{curl} \sigma(Y)\|_{2}\left\|p_{n}\right\|_{\infty}\left\|\sigma\left(p_{n}\right)\right\|_{2} \leq C\|\operatorname{curl} \sigma(Y)\|_{2}\left\|p_{n}\right\|_{H^{2}}^{2} \\
& \leq C\|Y\|_{\widetilde{W}}\left\|p_{n}\right\|_{W}^{2}
\end{aligned}
$$

and taking into account Lemma 3.6, we obtain

$$
\begin{aligned}
\left|\left(\operatorname{curl}\left(\sigma\left(Y \times p_{n}\right)\right), \mathbb{P} \sigma\left(p_{n}\right)\right)\right| & \leq\left|b\left(\sigma\left(p_{n}\right), Y, \mathbb{P} \sigma\left(p_{n}\right)\right)\right|+\mid b\left(Y, \mathbb{P} \sigma\left(p_{n}\right)-\sigma\left(p_{n}\right), \sigma\left(p_{n}\right) \mid\right. \\
& +\mid b\left(\sigma(Y), p_{n}, \mathbb{P} \sigma\left(p_{n}\right)\right)+b\left(p_{n}, \sigma(Y), \mathbb{P} \sigma\left(p_{n}\right) \mid\right) \\
& +\left|b\left(Y, p_{n}, \mathbb{P} \sigma\left(p_{n}\right)\right)-b\left(p_{n}, Y, \mathbb{P} \sigma\left(p_{n}\right)\right)\right| \\
& +\left|2 \alpha \sum_{i=1}^{2}\left(b\left(\frac{\partial p_{n}}{\partial x_{i}}, \frac{\partial Y}{\partial x_{i}}, \mathbb{P} \sigma\left(p_{n}\right)\right)-b\left(\frac{\partial Y}{\partial x_{i}}, \frac{\partial p_{n}}{\partial x_{i}}, \mathbb{P} \sigma\left(p_{n}\right)\right)\right)\right| \\
& \left.\leq\left\|\sigma\left(p_{n}\right)\right\|_{2}\|\nabla Y\|_{\infty} \| \mathbb{P} \sigma\left(p_{n}\right)\right) \|_{2} \\
& +\|Y\|_{\infty}\left\|\mathbb{P} \sigma\left(p_{n}\right)-\sigma\left(p_{n}\right)\right\|_{H^{1}}\left\|\sigma\left(p_{n}\right)\right\|_{2} \\
& +\|\sigma(Y)\|_{4}\left\|\nabla p_{n}\right\|_{4}\left\|\mathbb{P} \sigma\left(p_{n}\right)\right\|_{2}+\left\|p_{n}\right\|_{\infty}\|\nabla \sigma(Y)\|_{2}\left\|\mathbb{P} \sigma\left(p_{n}\right)\right\|_{2} \\
& +\|Y\|_{\infty}\left\|\nabla p_{n}\right\|_{2}\left\|\mathbb{P} \sigma\left(p_{n}\right)\right\|_{2}+\left\|p_{n}\right\|_{2}\|\nabla Y\|_{\infty}\left\|\mathbb{P} \sigma\left(p_{n}\right)\right\|_{2} \\
& +2 \alpha \sum_{i=1}^{2}\left[\left\|\frac{\partial p_{n}}{\partial x_{i}}\right\|_{4}\left\|\nabla\left(\frac{\partial Y}{\partial x_{i}}\right)\right\|_{4}\left\|\mathbb{P} \sigma\left(p_{n}\right)\right\|_{2}\right. \\
& \left.+\left\|\frac{\partial Y}{\partial x_{i}}\right\|_{\infty}\left\|\nabla\left(\frac{\partial p_{n}}{\partial x_{i}}\right)\right\|_{2}\left\|\mathbb{P} \sigma\left(p_{n}\right)\right\|_{2}\right] \\
& \leq C\|Y\|_{\widetilde{W}}\left\|p_{n}\right\|_{W}^{2} .
\end{aligned}
$$


By 3.26 we also have

$$
\left|\left(\operatorname{curl}\left(\sigma\left(Y \times p_{n}\right)\right), p_{n}\right)\right| \leq C\|Y\|_{\widetilde{W}}\left\|p_{n}\right\|_{V}^{2}
$$

As a consequence of estimates (7.9)-(7.11), there exists a fixed positive constant $C_{3}$, satisfying

$$
\begin{aligned}
2\left(f\left(p_{n}\right), \mathbb{P} \sigma\left(p_{n}\right)\right)+2\left(f\left(p_{n}\right), p_{n}\right) & \geq \frac{2 \nu}{\alpha}\left\|\mathbb{P} \sigma\left(p_{n}\right)\right\|_{W}^{2}+4 \nu\left\|D p_{n}\right\|_{2}^{2}-\frac{2 \nu}{\alpha}\left(p_{n}, \mathbb{P} \sigma\left(p_{n}\right)\right) \\
& -C_{3}\|Y\|_{\widetilde{W}}\left\|p_{n}\right\|_{W}^{2}-2\left(\nabla_{y} L(t, U, Y), \mathbb{P} \sigma\left(p_{n}\right)+p_{n}\right) .
\end{aligned}
$$

Reasoning as above in Lemma 5.2 , if we take the function $\xi_{3}(t)=e^{-C_{3} \int_{t}^{T}\|Y\|_{\widetilde{W}} d s}$, the Ito formula yields

$$
\begin{aligned}
d\left(\xi_{3}(t)\left\|p_{n}\right\|_{W}^{2}\right) & \geq \xi_{3}(t)\left(\frac{2 \nu}{\alpha}\left\|\mathbb{P} \sigma\left(p_{n}\right)\right\|_{2}^{2}+4 \nu\left\|D p_{n}\right\|_{2}^{2}-\frac{2 \nu}{\alpha}\left(p_{n}, \mathbb{P} \sigma\left(p_{n}\right)\right)\right) d t \\
& -2 \xi_{3}(t)\left(\nabla_{y} L(t, U, Y), \mathbb{P} \sigma\left(p_{n}\right)+p_{n}\right) d t \\
& -2 \xi_{3}(t)\left(\nabla_{y} G(t, Y)^{T} q_{n}, \mathbb{P} \sigma\left(p_{n}\right)+p_{n}\right) d t \\
& \left.+\xi_{3}(t)\left(2\left(\sigma\left(q_{n}\right), \mathbb{P} \sigma\left(p_{n}\right)+p_{n}\right)\right) d W_{t}+\left\|q_{n}\right\|_{W}^{2} d t\right)
\end{aligned}
$$

Integrating this inequality over the time interval $(t, T)$, we obtain

$$
\begin{aligned}
\xi_{3}(t)\left\|p_{n}(t)\right\|_{W}^{2} & +\int_{t}^{T} \xi_{3}\left(\frac{2 \nu}{\alpha}\left\|\mathbb{P} \sigma\left(p_{n}\right)\right\|_{2}^{2}+4 \nu\left\|D p_{n}\right\|_{2}^{2}+\left\|q_{n}\right\|_{W}^{2}\right) d s \\
& \left.\leq\left\|p_{n}(T)\right\|_{W}^{2}-2 \int_{t}^{T} \xi_{3}\left(\sigma\left(q_{n}\right), \mathbb{P} \sigma\left(p_{n}\right)+p_{n}\right)\right) d W_{s} \\
& +\frac{2 \nu}{\alpha} \int_{t}^{T} \xi_{3}\left(p_{n}, \mathbb{P} \sigma\left(p_{n}\right)\right) d s \\
& +2 \int_{t}^{T} \xi_{3}\left(\nabla_{y} L(s, U, Y), \mathbb{P} \sigma\left(p_{n}\right)+p_{n}\right) d s \\
& +2 \int_{t}^{T} \xi_{3}\left(\nabla_{y} G(s, Y)^{T} q_{n}, \mathbb{P} \sigma\left(p_{n}\right)+p_{n}\right) d s .
\end{aligned}
$$

We can apply Young's inequality $2 a b \leq \varepsilon a^{2}+b^{2} / \varepsilon$ to the last three terms. Therefore for an appropriate chosen of $\varepsilon$ in each of these three terms, accounting (4.1), we easily derive

$$
\begin{aligned}
\xi_{3}(t)\left\|p_{n}(t)\right\|_{W}^{2} & +\int_{t}^{T} \xi_{3}\left(\frac{\nu}{\alpha}\left\|\mathbb{P} \sigma\left(p_{n}\right)\right\|_{2}^{2}+4 \nu\left\|D p_{n}\right\|_{2}^{2}+\frac{1}{2}\left\|q_{n}\right\|_{W}^{2}\right) d s \\
& \leq\left\|p_{n}(T)\right\|_{W}^{2}+C \int_{t}^{T} \xi_{3}\left\|p_{n}\right\|_{W}^{2} d s+C \int_{t}^{T} \xi_{3}\left\|\nabla_{y} L(s, U, Y)\right\|_{2}^{2} d s \\
& \left.-2 \int_{t}^{T} \xi_{3}\left(\sigma\left(q_{n}\right), \mathbb{P} \sigma\left(p_{n}\right)+p_{n}\right)\right) d W_{s} .
\end{aligned}
$$

Let us define the stopping time

$$
\tau_{N}(\omega)=\sup \left\{t \in[0, T]: \min \left\{\left\|p_{n}(t)\right\|_{W},\left\|q_{n}\right\|_{W}\right\} \geq N\right\} \quad \text { for fixed } N \in \mathbb{N} .
$$

Let $\mathbf{1}_{\left[\tau_{N} \vee t, T\right]}$ be the characteristic function of the time interval $\left[\tau_{N} \vee t, T\right]$ for each $\omega \in \Omega$. The application of the expectation in (7.12) implies that the function $f(t)=$ 
$\mathbb{E} \mathbf{1}_{\left[\tau_{N} \vee t, T\right]} \xi_{3}(t)\left\|p_{n}(t)\right\|_{W}^{2} \quad$ fulfills the Gronwall type inequality

$$
\begin{aligned}
f(t) & +\int_{t}^{T} \mathbb{E} \mathbf{1}_{\left[\tau_{N} \vee t, T\right]} \xi_{3}(s)\left(\frac{\nu}{\alpha}\left\|\mathbb{P} \sigma\left(p_{n}\right)\right\|_{2}^{2}+4 \nu\left\|D p_{n}\right\|_{2}^{2}+\frac{1}{2}\left\|q_{n}\right\|_{W}^{2}\right) d s \\
& \leq \int_{t}^{T} f(s) d s+\mathbb{E}\left\|p_{n}(T)\right\|_{W}^{2}+C \mathbb{E} \int_{t}^{T} \xi_{3}(s)\left\|\nabla_{y} L(s, U, Y)\right\|_{2}^{2} d s,
\end{aligned}
$$

hence

$$
\begin{aligned}
f(t) & +\int_{t}^{T} \mathbb{E} \mathbf{1}_{\left[\tau_{N} \vee t, T\right]} \xi_{3}(s)\left(\frac{\nu}{\alpha}\left\|\mathbb{P} \sigma\left(p_{n}\right)\right\|_{2}^{2}+4 \nu\left\|D p_{n}\right\|_{2}^{2}+\frac{1}{2}\left\|q_{n}\right\|_{W}^{2}\right) d s \\
& \leq C\left(\mathbb{E}\|\nabla h(T)\|_{W}^{2}+\mathbb{E} \int_{t}^{T} \xi_{3}(s)\left\|\nabla_{y} L(s, U, Y)\right\|_{2}^{2} d s\right) \quad \text { for } t \in[0, T] .
\end{aligned}
$$

The Burkholder-Davis-Gundy inequality gives

$$
\begin{aligned}
2 \mathbb{E} \sup _{s \in\left[\tau_{N} \vee t, T\right]} \mid & \int_{s}^{T} \xi_{3}(r)\left(\sigma\left(q_{n}\right), \mathbb{P} \sigma\left(p_{n}\right)+p_{n}\right) d W_{r} \mid \\
& \leq 2 \mathbb{E}\left(\int_{\tau_{N} \vee t}^{T} \xi_{3}^{2}(s)\left|\left(q_{n}, \mathbb{P} \sigma\left(p_{n}\right)+p_{n}\right)\right|^{2} d s\right)^{\frac{1}{2}} \\
& \leq 2 \mathbb{E} \sup _{s \in\left[\tau_{N} \vee t, T\right]} \sqrt{\xi_{3}(s)}\left\|\mathbb{P} \sigma\left(p_{n}\right)+p_{n}\right\|_{2}\left(\int_{\tau_{N} \vee t}^{T} \xi_{3}(s)\left\|\sigma\left(q_{n}\right)\right\|_{2}^{2} d s\right)^{\frac{1}{2}} \\
& \leq \frac{1}{2} \mathbb{E} \sup _{s \in\left[\tau_{N} \vee t, T\right]} \xi_{3}(s)\left\|p_{n}\right\|_{W}^{2}+2 \mathbb{E} \int_{t}^{T} \xi_{3}(s)\left\|q_{n}\right\|_{W}^{2} d s .
\end{aligned}
$$

Therefore taking the supremum on the time interval $s \in\left[\tau_{N} \vee t, T\right]$ in (7.12), the expectation and applying inequalities (7.13)-(7.14), we see that the function $g(t)=\sup _{s \in\left[\tau_{N} \vee t, T\right]} \xi_{3}(s)\left\|p_{n}\right\|_{W}^{2}$ satisfies the Gronwall type inequality

$$
g(t) \leq C \int_{t}^{T} g(s) d s+C\left(\mathbb{E}\|\nabla h(T)\|_{W}^{2}+\mathbb{E} \int_{t}^{T} \xi_{3}(s)\left\|\nabla_{y} L(s, U, Y)\right\|_{2}^{2} d s\right),
$$

that implies

$$
\frac{1}{2} \mathbb{E} \sup _{s \in\left[\tau_{N} \vee t, T\right]} \xi_{3}(s)\left\|p_{n}(s)\right\|_{W}^{2} \leq C\left(\mathbb{E}\left\|p_{n}(T)\right\|_{W}^{2}+\mathbb{E} \int_{t}^{T} \xi_{3}(s)\left\|\nabla_{y} L(s, U, Y)\right\|_{2}^{2} d s\right) .
$$

Hence, combining this result with (7.13), we derive

$$
\begin{aligned}
\frac{1}{2} \mathbb{E} \sup _{s \in\left[\tau_{N} \vee t, T\right]} \xi_{3}(s)\left\|p_{n}(s)\right\|_{W}^{2} & +\int_{\tau_{N} \vee t}^{T} \xi_{3}(s)\left(\frac{\nu}{\alpha}\left\|\mathbb{P} \sigma\left(p_{n}\right)\right\|_{2}^{2}+4 \nu\left\|D p_{n}\right\|_{2}^{2}+\frac{1}{2}\left\|q_{n}\right\|_{W}^{2}\right) d s \\
& \leq C\left(\mathbb{E}\left\|p_{n}(T)\right\|_{W}^{2}+\mathbb{E} \int_{t}^{T} \xi_{3}(s)\left\|\nabla_{y} L(s, U, Y)\right\|_{2}^{2} d s\right) .
\end{aligned}
$$

Passing to the limit $N \rightarrow \infty$ in this last inequality, we deduce the claimed result.

As a direct consequence of Proposition 7.2 we obtain the following existence result. 
Theorem 7.3 There exists a unique solution

$$
(p, q) \in L^{\infty}(0, T ; W) \times L^{2}(0, T ; W) \quad P-\text { a.e. in } \Omega
$$

of the backward stochastic system (7.1). Moreover there exists a positive constant $C_{3}$, such that the pair $(p, q)$ satisfies the following estimate for a.e. $t \in(0, T)$

$$
\begin{aligned}
\frac{1}{2} \mathbb{E} \sup _{s \in[t, T]} \xi_{3}(s)\|p(s)\|_{W}^{2} & +\mathbb{E} \int_{t}^{T} \xi_{3}\left(\frac{\nu}{\alpha}\|\mathbb{P} \sigma(p)\|_{2}^{2}+4 \nu\|D p\|_{2}^{2}+\frac{1}{2}\|q\|_{W}^{2}\right) d s \\
& \leq C\left(\mathbb{E}\|\nabla h(Y(T))\|_{W}^{2}+\mathbb{E} \int_{t}^{T} \xi_{3}\left\|\nabla_{y} L(s, U, Y)\right\|_{2}^{2} d s\right)
\end{aligned}
$$

for the function

$$
\xi_{3}(t)=e^{-C_{3} \int_{t}^{T}\|Y\|_{\widetilde{W}} d s}
$$

Proof. By (4.5) we have

$$
\sup _{t \in[0, T]}\|Y(t)\|_{\widetilde{W}}^{2} \leq C(\omega) \quad \text { for all } \omega \in \Omega \backslash A, \quad \text { where } P(A)=0,
$$

that is, there exists a positive constant $K(\omega)$, dependent only on $\omega \in \Omega \backslash A$, satisfying

$$
0<K(\omega) \leq \xi_{3}(t) \leq 1 \quad \text { for all } \omega \in \Omega \backslash A, \quad t \in[0, T]
$$

By a priori estimates of Proposition 7.2 we have

$$
\mathbb{E} \sup _{t \in[0, T]} \xi_{3}(t)\left\|p_{n}(t)\right\|_{W}^{2} \leq C, \quad \mathbb{E} \int_{0}^{T} \xi_{3}(t)\left\|q_{n}(t)\right\|_{W}^{2} d t \leq C,
$$

hence using (7.15) there exists a subsequence of the pairs $\left(p_{n}, q_{n}\right)$, such that

$$
\begin{array}{ll}
S_{n}=\sqrt{\xi_{3}} p_{n} \rightarrow S=\sqrt{\xi_{3}} p & \text { *-weakly in } L^{2}\left(\Omega, L^{\infty}(0, T ; W)\right), \\
Q_{n}=\sqrt{\xi_{3}} q_{n} \rightarrow Q=\sqrt{\xi_{3}} q & \text { weakly in } L^{2}\left(\Omega, L^{2}(0, T ; W)\right) .
\end{array}
$$

Moreover the pair $\left(p_{n}, q_{n}\right)$ solves

$$
\begin{aligned}
(\sigma(\nabla h(Y(T))), \phi)-\left(\sigma\left(p_{n}(t)\right), \phi\right) & =\int_{t}^{T}\left\{2 \nu\left(D p_{n}(s), D \phi\right)\right. \\
& -b\left(\phi, p_{n}(s), \sigma(Y(s))\right)+b\left(p_{n}(s), \phi, \sigma(Y(s))\right) \\
& +b\left(p_{n}(s), Y(s), \sigma(\phi(s))\right)-b\left(Y(s), p_{n}(s), \sigma(\phi(s))\right) \\
& \left.+\int_{t}^{T}\left(\nabla_{y} L(s, U, Y)+\nabla_{y} G(s, Y)^{T} q_{n}, \phi\right)\right\} d s \\
& +\int_{t}^{T}\left(\sigma\left(q_{n}(s)\right), \phi\right) d W_{s} \quad \text { for each } \phi \in V
\end{aligned}
$$

therefore we can apply similar arguments as in the proof of Theorem 5.3 and demonstrate that the pair $(p, q)$ satisfies equation (7.2). 


\subsection{Exponential integrability for the solution of equation (2.1)}

As in the previous sections we consider that the data $U, Y_{0}$ satisfy assumptions (4.4) and denote the unique solution of the stochastic differential equation (4.3) by

$$
Y \in L^{p}\left(\Omega, L^{\infty}(0, T ; V)\right) \cap L^{2}\left(\Omega, L^{\infty}(0, T ; \widetilde{W})\right),
$$

satisfying estimates (4.5). In what follows, we assume an additional assumptions on the data in order to improve the integrability of the stochastic process $Y$. We will show that under these additional assumptions, the stochastic process $Y$ is exponential integrable.

Let $U$ be a distributed mechanical force belonging to the admissible set $\mathcal{U}_{a d}^{b}$, which is defined as the set of all adapted stochastic processes

$$
U=U(\omega, t) \in L^{\infty}\left(\Omega, L^{2}(0, T ; V)\right) \cap L^{p}\left(\Omega, L^{p}\left(0, T ; H^{1}(\mathcal{O})\right)\right) \quad \text { for some } 4 \leq p<\infty,
$$

uniformly bounded in $L^{2}(0, T ; V)$, this means that there exists a positive constant $M$, independent of $\omega$, such that

$$
\int_{0}^{T}\|U(\omega, t)\|_{V}^{2} d t \leq M \quad \text { for } P-\text { a.e. } \omega \in \Omega
$$

We also introduce additional hypothesis on the diffusion operator $G$, namely $G$ is bounded by a positive constant $L$ in the space $V$

$$
\|G(t, y)\|_{V}^{2} \leq L \quad \text { for all } t \in[0, T], \quad y \in V .
$$

Let us set $C_{\max }=\max \left\{C_{1}, C_{2}, C_{3}\right\}$, where $C_{i}, i=1,2,3$, are the constants defined in Propositions 5.2 and 7.2 , and introduce the following two conditions:

the first condition is given by

$$
A=\frac{1}{2 \theta_{1}} \geq C_{\max }
$$

and the second one reads as

$$
\text { the domain } \mathcal{O} \text { is non axisymmetric and } B=\frac{\gamma_{2}^{2}}{2 \theta_{2}} \geq C_{\max } .
$$

Here the constants $\theta_{1}, \theta_{2}, \gamma_{1}, \bar{\gamma}_{1}, \gamma_{2}$ and $\tau$ are defined by

$$
\begin{aligned}
& \theta_{1}=4 L \tau^{2}\left(1+\gamma_{1}^{2}\right), \quad \theta_{2}=2 L e^{\varepsilon T}\left(1+2 \bar{\gamma}_{1}^{2}\right), \\
& \gamma_{1}=\frac{C_{*} \nu}{\alpha} \tau, \quad \bar{\gamma}_{1}=\frac{K_{*}}{4 \alpha} \tau, \quad \gamma_{2}=\frac{C_{* *} \nu}{\alpha} \quad \text { and } \quad \tau=T e^{\varepsilon T},
\end{aligned}
$$

with the constants $C_{*}, K_{*}$ and $C_{* *}$ introduced in (3.6), (3.7) and (3.12).

Now, we consider the strong solution $Y$ of the state system (2.1) which exists by Theorem 4.2, and our purpose is to show the exponential integrability of the state process $Y$. Let us mention that the two main arguments to show this result rely on the structure of equation (2.1) ${ }_{1}$ for $Y$, and on the martingale property of the exponential process that appears in the right hand side of inequalities (7.38) $-(7.39)$. 
Proposition 7.4 Assume that $U \in \mathcal{U}_{a d}^{b}$, satisfies 7.18-17.19) and the initial condition

$$
Y_{0} \in L^{p}(\Omega, V) \cap L^{\infty}(\Omega, \widetilde{W})
$$

Also we admit that condition (7.20) and one of two conditions (7.21) or (7.22) hold, then there exists a positive constant $C$, such that the following estimate is valid

$$
\mathbb{E} \exp \left\{C_{\max } \int_{0}^{t}\|Y(s)\|_{\widetilde{W}}^{2} d s\right\} \leq C \quad \text { for a.e. } t \in(0, T) .
$$

Proof. 1st step: Estimates for $Y$ in the space $V$.

Let $\widetilde{G}$ be the solution of (3.8) for $f=G(t, Y)$. Using the fact that $\widetilde{G}$ solves the elliptic type problem (3.8) for $f=G(t, Y)$, (3.13) and assumption (7.20), we have

$$
\|\widetilde{G}\|_{V}^{2} \leq\|G(t, Y)\|_{2}^{2} \leq L
$$

Applying the operator $(I-\alpha \mathbb{P} \Delta)^{-1}$ to equation $(2.1)_{1}$, we deduce a stochastic differential equation for $Y$, then with the help of Itô's formula, as it was done in the article [9], we obtain

$$
d\|Y\|_{V}^{2}=2\left(-2 \nu\|D Y\|_{2}^{2}+(U, Y)\right) d t+2(G(t, Y), Y) d W_{t}+\|\widetilde{G}\|_{V}^{2} d t
$$

Using Young's inequality $2 a b \leq \frac{a^{2}}{\varepsilon}+\varepsilon b^{2}$ and integrating over the time interval $(0, t)$, we obtain

$$
\|Y(t)\|_{V}^{2}+4 \nu \int_{0}^{t}\|D Y\|_{2}^{2} d s \leq \varepsilon \int_{0}^{t}\|Y\|_{2}^{2} d s+\left(C_{\varepsilon}+g_{1}(t)\right),
$$

where

$$
\begin{aligned}
C_{\varepsilon} & =\|Y(0)\|_{V}^{2}+\frac{1}{\varepsilon} \int_{0}^{T}\|U\|_{2}^{2} d t+L T \\
g_{1}(t) & =\int_{0}^{t} f_{1}(s) d W_{s}, \quad f_{1}(s)=2(G(s, Y), Y) .
\end{aligned}
$$

Taking $z(t)=\int_{0}^{t}\|Y\|_{V}^{2} d s$, expression (7.26) gives the differential inequality $z^{\prime} \leq \varepsilon z+\left(C_{\varepsilon}+g_{1}(t)\right)$, which can be integrated by Gronwall's lemma. Hence

$$
\begin{aligned}
\int_{0}^{t}\|Y\|_{V}^{2} d s & =z(t) \leq e^{\varepsilon t} \int_{0}^{t} e^{-\varepsilon s}\left(C_{\varepsilon}+g_{1}(s)\right) d s \\
& =C_{\varepsilon}\left(\frac{e^{\varepsilon t}-1}{\varepsilon}\right)+e^{\varepsilon t} \int_{0}^{t} e^{-\varepsilon s} g_{1}(s) d s
\end{aligned}
$$

Since the inequality $\left|\frac{e^{x}-1}{x}\right| \leq e^{|x|}$, we have $\left|\frac{e^{\varepsilon t}-1}{\varepsilon}\right| \leq t e^{\varepsilon t} \leq T e^{\varepsilon T}$. Applying Fubini's theorem, we derive

$$
e^{\varepsilon t} \int_{0}^{t} e^{-\varepsilon s} g_{1}(s) d s=\int_{0}^{t}\left(\frac{e^{\varepsilon(t-s)}-1}{\varepsilon}\right) f_{1}(s) d W_{s} .
$$

Hence

$$
\int_{0}^{t}\|Y\|_{V}^{2} d s \leq C_{\varepsilon} T e^{\varepsilon T}+\int_{0}^{t}\left(\frac{e^{\varepsilon(t-s)}-1}{\varepsilon}\right) f_{1}(s) d W_{s} .
$$


Now, using (7.27) to estimate the right hand side of (7.26), we deduce

$$
\begin{aligned}
& \|Y(t)\|_{V}^{2}+4 \nu \int_{0}^{t}\|D Y\|_{2}^{2} d s \leq \varepsilon \int_{0}^{t}\|Y\|_{2}^{2} d s+\left(C_{\varepsilon}+g_{1}(t)\right) \\
& \leq \varepsilon\left[e^{\varepsilon t} \int_{0}^{t} e^{-\varepsilon s}\left(C_{\varepsilon}+g_{1}(s)\right) d s\right]+\left(C_{\varepsilon}+g_{1}(t)\right) \\
& =C_{\varepsilon} e^{\varepsilon t}+\varepsilon e^{\varepsilon t} \int_{0}^{t} e^{-\varepsilon s} g_{1}(s) d s+g_{1}(t) \\
& =C_{\varepsilon} e^{\varepsilon t}+\int_{0}^{t} e^{\varepsilon(t-s)} f_{1}(s) d W_{s}
\end{aligned}
$$

by (7.28). Therefore

$$
\|Y(t)\|_{V}^{2}+4 \nu \int_{0}^{t}\|D Y\|_{2}^{2} d s \leq C_{\varepsilon} e^{\varepsilon t}+\int_{0}^{t} e^{\varepsilon(t-s)} f_{1}(s) d W_{s} .
$$

2nd step: Estimates for curl $Y$ in the space $L^{2}$. Using (3.6) and (7.29), we obtain

$$
\frac{\nu}{\alpha} \int_{0}^{t}\|\operatorname{curl} Y\|_{2}^{2} d s \leq \frac{C_{*} \nu}{\alpha}\left\{C_{\varepsilon} T e^{\varepsilon T}+\int_{0}^{t}\left(\frac{e^{\varepsilon(t-s)}-1}{\varepsilon}\right) f_{1}(s) d W_{s}\right\} .
$$

Under the assumption that the domain $\mathcal{O}$ is not axisymmetric, we may apply inequality (3.7), in order to deduce as alternative estimate for $\operatorname{curl} Y$, namely, we have

$$
\frac{\nu}{\alpha} \int_{0}^{t}\|\operatorname{curl} Y\|_{2}^{2} d s \leq \frac{\nu}{\alpha} \int_{0}^{t}\|Y\|_{H^{1}}^{2} d s \leq \frac{K_{*} \nu}{\alpha} \int_{0}^{t}\|D Y\|_{2}^{2} d s
$$

and considering (7.30), we obtain

$$
\frac{\nu}{\alpha} \int_{0}^{t}\|\operatorname{curl} Y\|_{2}^{2} d s \leq \frac{K_{*}}{4 \alpha}\left\{C_{\varepsilon} e^{\varepsilon T}+\int_{0}^{t} e^{\varepsilon(t-s)} f_{1}(s) d W_{s}\right\} .
$$

3d step: Two estimates for $\operatorname{curl} \sigma(Y)$ in the space $L^{2}$. Applying the operator curl to equation $(2.1)_{1}$, we deduce

$$
\begin{aligned}
d(\operatorname{curl} \sigma(Y)) & =\frac{\nu}{\alpha}(-\operatorname{curl} \sigma(Y)+\operatorname{curl} Y) d t+\operatorname{curl} U d t \\
& -(Y \cdot \nabla) \operatorname{curl} \sigma(Y) d t+\operatorname{curl} G(t, Y) d W_{t} .
\end{aligned}
$$

The Itô's formula yields

$$
\begin{aligned}
& d\|\operatorname{curl} \sigma(Y)\|_{2}^{2}=\frac{2 \nu}{\alpha}(-\operatorname{curl} \sigma(Y)+\operatorname{curl} Y, \operatorname{curl} \sigma(Y)) d t+2(\operatorname{curl} U, \operatorname{curl} \sigma(Y)) d t \\
& -2((Y \cdot \nabla) \operatorname{curl} \sigma(Y), \operatorname{curl} \sigma(Y)) d t+2(\operatorname{curl} G(t, Y), \operatorname{curl} \sigma(Y)) d W_{t}+\|\operatorname{curl} G(t, Y)\|_{2}^{2} d t .
\end{aligned}
$$

Since

$$
((Y \cdot \nabla) \operatorname{curl} \sigma(Y), \operatorname{curl} \sigma(Y))=0
$$


we derive

$$
\begin{aligned}
d\|\operatorname{curl} \sigma(Y)\|_{2}^{2} & =\frac{2 \nu}{\alpha}(-\operatorname{curl} \sigma(Y)+\operatorname{curl} Y, \operatorname{curl} \sigma(Y)) d t+2(\operatorname{curl} U, \operatorname{curl} \sigma(Y)) d t \\
& +2(\operatorname{curl} G(t, Y), \operatorname{curl} \sigma(Y)) d W_{t}+\|\operatorname{curl} G(t, Y)\|_{2}^{2} d t .
\end{aligned}
$$

From assumption (7.20) we have

$$
\|\operatorname{curl} G(t, Y)\|_{2}^{2} \leq C\|G(t, Y)\|_{V}^{2} \leq C L,
$$

in addition, the relation $2(-y+x) y \leq-y^{2}+x^{2}, \forall x, y \in \mathbb{R}$, gives

$$
2(-\operatorname{curl} \sigma(Y)+\operatorname{curl} Y, \operatorname{curl} \sigma(Y)) \leq-\|\operatorname{curl} \sigma(Y)\|_{2}^{2}+\|\operatorname{curl} Y\|_{2}^{2} .
$$

Hence, introducing these estimates in (17.33) and integrating over $[0, t]$, we obtain

$$
\begin{aligned}
\|\operatorname{curl} \sigma(Y)\|_{2}^{2} & +\frac{\nu}{\alpha} \int_{0}^{t}\|\operatorname{curl} \sigma(Y)\|_{2}^{2} d s \leq\|\operatorname{curl} \sigma(Y(0))\|_{2}^{2}+\frac{\nu}{\alpha} \int_{0}^{t}\|\operatorname{curl} Y\|_{2}^{2} d s+C L T \\
& +2 \int_{0}^{t}(\operatorname{curl} U, \operatorname{curl} \sigma(Y)) d s+2 \int_{0}^{t}(\operatorname{curl} G(s, Y), \operatorname{curl} \sigma(Y)) d W_{s} .
\end{aligned}
$$

Therefore, if we use Young's inequality $2 a b \leq \frac{a^{2}}{\varepsilon}+\varepsilon b^{2}$ and (7.31), we deduce

$$
\|\operatorname{curl} \sigma(Y)\|_{2}^{2}+\frac{\nu}{\alpha} \int_{0}^{t}\|\operatorname{curl} \sigma(Y)\|_{2}^{2} d t \leq \varepsilon \int_{0}^{t}\|\operatorname{curl} \sigma(Y)\|^{2} d s+\left(\widetilde{C}_{\varepsilon}+g_{2}(t)\right),
$$

where

$$
\begin{aligned}
\widetilde{C}_{\varepsilon} & =\frac{1}{\varepsilon} \int_{0}^{T}\|\operatorname{curl} U\|_{2}^{2} d s+\|\operatorname{curl} \sigma(Y(0))\|_{2}^{2}+C L T+\frac{C_{*} \nu}{\alpha} T e^{\varepsilon T}, \\
g_{2}(t) & =\int_{0}^{t} f_{2}(s) d W_{s}, \\
f_{2}(s) & =2(\operatorname{curl} G(s, Y), \operatorname{curl} \sigma(Y))+\frac{C_{*} \nu}{\alpha}\left(\frac{e^{\varepsilon(t-s)}-1}{\varepsilon}\right) 2(G(s, Y), Y) .
\end{aligned}
$$

On the other hand, due to (7.32) we also have

$$
\|\operatorname{curl} \sigma(Y)\|_{2}^{2}+\frac{\nu}{\alpha} \int_{0}^{t}\|\operatorname{curl} \sigma(Y)\|_{2}^{2} d t \leq \varepsilon \int_{0}^{t}\|\operatorname{curl} \sigma(Y)\|^{2} d s+\left(\bar{C}_{\varepsilon}+\bar{g}_{2}(t)\right),
$$

where

$$
\begin{aligned}
\bar{C}_{\varepsilon} & =\frac{1}{\varepsilon} \int_{0}^{T}\|\operatorname{curl} U\|_{2}^{2} d s+\|\operatorname{curl} \sigma(Y(0))\|_{2}^{2}+C L T+\frac{K_{*}}{4 \alpha} e^{\varepsilon T}, \\
\bar{g}_{2}(t) & =\int_{0}^{t} \bar{f}_{2}(s) d W_{s}, \\
\bar{f}_{2}(s) & =2(\operatorname{curl} G(s, Y), \operatorname{curl} \sigma(Y))+\frac{K_{*}}{2 \alpha} e^{\varepsilon(t-s)}(G(s, Y), Y) .
\end{aligned}
$$

Now, we proceed as in the 1st step, defining $z(t)=\int_{0}^{t}\|\operatorname{curl} \sigma(Y)\|_{2}^{2} d s$. In the former case, we get the inequality $z^{\prime} \leq \varepsilon z+\left(\widetilde{C}_{\varepsilon}+g_{2}(t)\right)$, which may be integrated to obtain

$$
\int_{0}^{t}\|\operatorname{curl} \sigma(Y)\|_{2}^{2} d s=z(t) \leq \widetilde{C}_{\varepsilon} T e^{\varepsilon T}+\int_{0}^{t}\left(\frac{e^{\varepsilon(t-s)}-1}{\varepsilon}\right) f_{2}(s) d W_{s} .
$$


Applying the same reasoning, the second situation gives

$$
\|\operatorname{curl} \sigma(Y)\|_{2}^{2}+\frac{\nu}{\alpha} \int_{0}^{t}\|\operatorname{curl} \sigma(Y)\|_{2}^{2} d s \leq \bar{C}_{\varepsilon} e^{\varepsilon t}+\int_{0}^{t} e^{\varepsilon(t-s)} \bar{f}_{2}(s) d W_{s} .
$$

For details we refer to the deduction of inequalities (7.29) and (17.30).

4th step: Two exponential estimates for $Y$. Taking the sum of inequalities (7.29) and (7.34) we get

$$
\int_{0}^{t}\|Y\|_{\widetilde{W}}^{2} d s \leq\left(C_{\varepsilon}+\widetilde{C}_{\varepsilon}\right) T e^{\varepsilon T}+\int_{0}^{t}\left(\frac{e^{\varepsilon(t-s)}-1}{\varepsilon}\right)\left[f_{1}(s)+f_{2}(s)\right] d W_{s}
$$

and the sum of inequalities (7.30) and (7.35) yields

$$
\frac{\nu}{\alpha} \int_{0}^{t}\left(2 \alpha\|D Y\|_{2}^{2}+\|\operatorname{curl} \sigma(Y)\|_{2}^{2}\right) d s \leq\left(\frac{1}{2} C_{\varepsilon}+\bar{C}_{\varepsilon}\right) e^{\varepsilon t}+\int_{0}^{t} e^{\varepsilon(t-s)}\left[\frac{1}{2} f_{1}(s)+\bar{f}_{2}(s)\right] d W_{s},
$$

then applying (3.12), we derive

$$
\frac{C_{* *} \nu}{\alpha} \int_{0}^{t}\|Y\|_{\widetilde{W}}^{2} d s \leq \frac{1}{2}\left(C_{\varepsilon}+\bar{C}_{\varepsilon}\right) e^{\varepsilon t}+\int_{0}^{t} e^{\varepsilon(t-s)}\left[\frac{1}{2} f_{1}(s)+\bar{f}_{2}(s)\right] d W_{s} .
$$

Let us denote by

$$
Z_{1}(s)=\left(\frac{e^{\varepsilon(t-s)}-1}{\varepsilon}\right)\left[f_{1}(s)+f_{2}(s)\right], \quad Z_{2}(s)=e^{\varepsilon(t-s)}\left[\frac{1}{2} f_{1}(s)+\bar{f}_{2}(s)\right]
$$

and the constants

$$
\left.H_{1}=\exp \left(\left(C_{\varepsilon}+\widetilde{C}_{\varepsilon}\right) T e^{\varepsilon T}\right), \quad H_{2}=\exp \left(\frac{1}{2} C_{\varepsilon}+\bar{C}_{\varepsilon}\right) e^{\varepsilon T}\right) \quad \text { for any fixed } \varepsilon>0 .
$$

Therefore if we multiply (7.36) and (7.37) by $\lambda$, applying the exponential function and the expectation, we deduce

$$
\begin{aligned}
& \mathbb{E} \exp \left\{\left(\lambda-\frac{1}{2} \lambda^{2} \theta_{1}\right) \int_{0}^{t}\|Y\|_{\widetilde{W}}^{2} d s\right\} \\
& \leq \mathbb{E} \exp \left\{\lambda \int_{0}^{t}\|Y\|_{\widetilde{W}}^{2} d s-\frac{\lambda^{2}}{2} \int_{0}^{t} Z_{1}(s)^{2} d s\right\} \\
& \leq H_{1} \mathbb{E} \exp \left\{\int_{0}^{t} \lambda Z_{1}(s) d W_{s}-\frac{1}{2} \int_{0}^{t}\left(\lambda Z_{1}(s)\right)^{2} d s\right\}
\end{aligned}
$$

and

$$
\begin{aligned}
& \mathbb{E} \exp \left\{\left(\gamma_{2} \lambda-\frac{1}{2} \lambda^{2} \theta_{2}\right) \int_{0}^{t}\|Y\|_{\widetilde{W}}^{2} d s\right\} \\
& \leq \mathbb{E} \exp \left\{\lambda \int_{0}^{t}\|Y\|_{\widetilde{W}}^{2} d s-\frac{\lambda^{2}}{2} \int_{0}^{t} Z_{2}(s)^{2} d s\right\} \\
& \leq H_{2} \mathbb{E} \exp \left\{\int_{0}^{t} \lambda Z_{2}(s) d W_{s}-\frac{1}{2} \int_{0}^{t}\left(\lambda Z_{2}(s)\right)^{2} d s\right\}
\end{aligned}
$$


since $G(t, y), U$ and the initial condition $Y_{0}$ satisfy (7.20), (7.19) and (7.24), respectively. Here the constants $\theta_{1}, \theta_{2}, \gamma_{1}, \gamma_{2}$ and $\tau$ are defined by (7.23).

Taking into account that the expectations of the right hand sides in (7.38) and in (7.39) are equal to 1 , we derive

$$
\mathbb{E} \exp \left\{\left(\lambda-\frac{\lambda^{2}}{2} \theta_{1}\right) \int_{0}^{t}\|Y\|_{\widetilde{W}}^{2} d s\right\} \leq H_{1}
$$

and

$$
\mathbb{E} \exp \left\{\left(\gamma_{2} \lambda-\frac{\lambda^{2}}{2} \theta_{2}\right) \int_{0}^{t}\|Y\|_{\widetilde{W}}^{2} d s\right\} \leq H_{2} .
$$

Hence, we derive estimate (7.25), since one of conditions (7.21), (7.22) holds and

$$
A=\frac{1}{2 \theta_{1}}=\max _{\lambda}\left(\lambda-\frac{\lambda^{2}}{2} \theta_{1}\right) \quad \text { and } \quad B=\frac{\gamma_{2}^{2}}{2 \theta_{2}}=\max _{\lambda}\left(\gamma_{2} \lambda-\frac{\lambda^{2}}{2} \theta_{2}\right) .
$$

\subsection{Duality property}

In the next proposition we prove that the functions $Z_{n}$, constructed in Proposition 5.2, and $\left(p_{n}, q_{n}\right)$, constructed in Proposition 7.2, satisfy the so-called duality property.

Proposition 7.5 The solution $Z_{n}$ of system (7.4) and the solution $\left(p_{n}, q_{n}\right)$ of system 7.2) satisfy the duality equality

$$
\mathbb{E}\left(\sigma\left(Z_{n}(T)\right), \nabla h\left(Y_{T}\right)\right)+\mathbb{E} \int_{0}^{T}\left(\nabla_{y} L(t, U, Y), Z_{n}\right) d t=\mathbb{E} \int_{0}^{T}\left(\Psi, p_{n}\right) d t
$$

for any $\Psi \in L^{2}(\Omega \times(0, T) \times \mathcal{O})$.

Proof. Setting $\phi=h_{i}$ in equation (7.4), we obtain

$$
d\left(p_{n}, h_{i}\right)_{V}=\left(\tilde{f}\left(p_{n}\right), h_{i}\right) d t-\left(R\left(q_{n}\right), h_{i}\right) d t+\left(\sigma\left(q_{n}\right), h_{i}\right) d W_{t}
$$

for

$$
\begin{aligned}
\tilde{f}\left(p_{n}\right) & =-\nu \Delta p_{n}-\operatorname{curl} \sigma(Y) \times p_{n}+\operatorname{curl}\left(\sigma\left(Y \times p_{n}\right)\right), \\
R\left(q_{n}\right) & =\nabla_{y} L(t, U, Y)+\nabla_{y} G(t, Y)^{T} q_{n} .
\end{aligned}
$$

Let us remember that the solution

$$
Z_{n}(t)=\sum_{i=1}^{n} \zeta_{i}(t) h_{i}
$$


solves the stochastic differential equation (5.5). Therefore multiplying (7.41) by $\zeta_{i}(t)$ and summing over $i=1, \ldots, n$, we obtain

$$
\left(d p_{n}, Z_{n}\right)_{V}=\left(\tilde{f}\left(p_{n}\right), Z_{n}\right) d t-\left(R\left(q_{n}\right), Z_{n}\right) d t+\left(q_{n}, Z_{n}\right)_{V} d W_{t} .
$$

Using property (3.18) we get the following identity

$$
\begin{aligned}
\left(d p_{n}, Z_{n}\right)_{V} & =\left\{2 \nu\left(D p_{n}(t), D Z_{n}\right) d x\right. \\
& -b\left(Z_{n}, p_{n}, \sigma(Y)\right)+b\left(p_{n}, Z_{n}, \sigma(Y)\right) \\
& +b\left(p_{n}, Y, \sigma\left(Z_{n}\right)\right)-b\left(Y, p_{n}, \sigma\left(Z_{n}\right)\right) \\
& \left.-\left(\nabla_{y} L(t, U, Y)+\nabla_{y} G(t, Y)^{T} q_{n}, Z_{n}\right)\right\} d t \\
& +\left(q_{n}, \sigma\left(Z_{n}\right)\right) d W_{t} .
\end{aligned}
$$

By another hand since $Z_{n}$ is the solution of (5.5), then setting $\phi=h_{i}$ in this equation, we get

$$
d\left(Z_{n}, h_{i}\right)_{V}=\left(f\left(Z_{n}\right), h_{i}\right) d t+\left(\nabla_{y} G(t, Y) Z_{n}, h_{i}\right) d W_{t},
$$

where $f\left(Z_{n}\right)=\nu \Delta Z_{n}-\operatorname{curl} \sigma\left(Z_{n}\right) \times Y-\operatorname{curl} \sigma(Y) \times Z_{n}+\Psi$. Recalling that $p_{n}$ is defined by (7.3), if we multiply the last equality by $\mathfrak{p}_{i}(t)$ and sum over $i=1, \ldots, n$, we deduce

$$
\left(d Z_{n}, p_{n}\right)_{V}=\left(f\left(Z_{n}\right), p_{n}\right) d t+\left(\nabla_{y} G(t, Y) Z_{n}, p_{n}\right) d W_{t} .
$$

Applying identities (3.18) and (3.25), we derive

$$
\begin{aligned}
\left(d Z_{n}, p_{n}\right)_{V}= & \left\{-2 \nu\left(D p_{n}(t), D Z_{n}\right)\right. \\
& -b\left(p_{n}, Y, \sigma\left(Z_{n}\right)\right)+b\left(Y, p_{n}, \sigma\left(Z_{n}\right)\right) \\
& -b\left(p_{n}, Z_{n}, \sigma(Y)\right)+b\left(Z_{n}, p_{n}, \sigma(Y)\right) \\
& \left.+\left(\Psi, p_{n}\right)\right\} d t+\left(\nabla_{y} G(t, Y) Z_{n}, p_{n}\right) d W_{t} .
\end{aligned}
$$

Hence Ito's formula gives

$$
\begin{aligned}
d\left(Z_{n}, p_{n}\right)_{V}= & \left(d Z_{n}, p_{n}\right)_{V}+\left(Z_{n}, d p_{n}\right)_{V}+\left(d Z_{n}, d p_{n}\right)_{V} \\
= & {\left[-\left(\nabla_{y} L(t, U, Y), Z_{n}\right)+\left(\Psi, p_{n}\right)\right] d t } \\
& +\left[\left(q_{n}, \sigma\left(Z_{n}\right)\right)+\left(\nabla_{y} G(t, Y) Z_{n}, p_{n}\right)\right] d W_{t},
\end{aligned}
$$

combining formulaes (7.42) and (7.43). The integration of the last identity over the time interval $[0, T]$ gives

$$
\begin{aligned}
\left(Z_{n}(T), p_{n}(T)\right)_{V} & =\int_{0}^{T}\left(\Psi, p_{n}\right) d t-\left(\nabla_{y} L(t, U, Y), Z_{n}\right) d t \\
& +\int_{0}^{T}\left[\left(q_{n}, \sigma\left(Z_{n}\right)\right)+\left(\nabla_{y} G(t, Y) Z_{n}, p_{n}\right)\right] d W_{t} .
\end{aligned}
$$

Finally taking the expectation, we conclude that the pair $\left(p_{n}, q_{n}\right)$ satisfies relation (7.40).

In the next theorem we prove that $Z$, constructed in Theorem [5.3, and $(p, q)$, constructed in Theorem 7.3 satisfy the duality condition. 
Theorem 7.6 Under assumptions of Proposition 7.4, the solution

$$
Z \in L^{2}\left(\Omega, L^{\infty}(0, T ; W)\right)
$$

of system (5.2) and the solution $(p, q) \in L^{2}\left(\Omega, L^{\infty}(0, T ; W)\right) \times L^{2}\left(\Omega, L^{2}(0, T ; W)\right)$ of the backward stochastic equation (7.1) satisfy the following duality property

$$
\mathbb{E} \int_{0}^{T}\left(\nabla_{y} L(t, U, Y), Z\right) d t+\mathbb{E}\left(\sigma(Z(T)), \nabla h\left(Y_{T}\right)\right)=\mathbb{E} \int_{0}^{T}(\Psi, p) d t
$$

for any $\Psi \in L^{2}(\Omega \times(0, T) \times \mathcal{O})$.

Proof. Firstly let us show that for subsequences of $\left\{Z_{n}\right\}$ and $\left\{\left(p_{n}, q_{n}\right)\right\}$ we have

$$
\begin{aligned}
\mathbb{E} \int_{0}^{T}\left(\nabla_{y} L(t, U, Y), Z_{n}\right) d t & \rightarrow \mathbb{E} \int_{0}^{T}\left(\nabla_{y} L(t, U, Y), Z\right) d t, \\
\mathbb{E}\left(\sigma\left(Z_{n}(T)\right), \nabla h\left(Y_{T}\right)\right) & \rightarrow \mathbb{E}\left(\sigma(Z(T)), \nabla h\left(Y_{T}\right)\right), \\
\mathbb{E} \int_{0}^{T}\left(\Psi, p_{n}\right) d t & \rightarrow \mathbb{E} \int_{0}^{T}(\Psi, p) d t \quad \text { as } n \rightarrow \infty
\end{aligned}
$$

with $Z$ and the pair $\{(p, q)\}$ defined in (5.20), (7.16).

By assumptions (6.26) we know that $\nabla_{y} L(t, U, Y) \in L^{2}(\Omega \times(0, T) \times \mathcal{O})$. Due to the absolute continuity of the Lebesgue integral, for any fixed $\varepsilon>0$, there exists $\lambda=\lambda(\varepsilon)>0$ such that

$$
\mathbb{E} \int_{0}^{T}\left\|\chi \nabla_{y} L(t, U, Y)\right\|^{2} d t<\varepsilon
$$

where $\chi$ is the characteristic function of the set $\left\{(t, x, \omega):\left|\nabla_{y} L(t, U, Y)\right|>\lambda\right\}$. Hence

$$
\begin{aligned}
\left|\mathbb{E} \int_{0}^{T}\right|\left(\chi \nabla_{y} L(t, U, Y), Z_{n}-Z\right) d t \mid & \leq\left(\mathbb{E} \int_{0}^{T}\left\|\chi \nabla_{y} L(t, U, Y)\right\|^{2} d t\right)^{1 / 4} \times\left(\mathbb{E} \int_{0}^{T} \xi_{1}^{-2}(t) d t\right)^{1 / 4} \\
& \times\left(\mathbb{E} \int_{0}^{T} \xi_{1}(t)\left(\left\|Z_{n}\right\|^{2}+\|Z\|^{2}\right) d t\right)^{1 / 4} \leq C \varepsilon \quad(7.46)
\end{aligned}
$$

as a direct consequence of (5.6), (5.20) and (7.25).

Since the function $(1-\chi) \nabla_{y} L(t, U, Y)$ is bounded by $\lambda$, therefore, using again (5.6), (5.20) and (7.25), we deduce

$$
\mathbb{E} \int_{0}^{T}\left((1-\chi) \nabla_{y} L(t, U, Y), Z_{n}-Z\right) d t \rightarrow 0 \quad \text { as } n \rightarrow \infty .
$$

Combining (7.46) and (7.47), we derive (7.45) 1 . The justification of other two limits in (7.45) can be done by a similar way.

The function $Z_{n}$ and the pair $\left(p_{n}, q_{n}\right)$ fulfill (7.40), therefore taking the limit transition in this equality we show that $Z$ and the pair $(p, q)$ satisfy equality (7.44). 


\section{Existence and optimality condition}

Let us state the main result of this article. Which essentially says that the optimal control $U \in \mathcal{U}_{a d}^{b}$ exists and may be determined through the optimality condition.

Let us recall the assumptions made along the article. We assume $U \in \mathcal{U}_{a d}^{b}$ satisfying (7.18) (7.19) and the initial condition

$$
Y_{0} \in L^{p}(\Omega, V) \cap L^{\infty}(\Omega, \widetilde{W})
$$

moreover, we admit that (7.20) and one of conditions (7.21), (7.22) hold. The functional $J$ satisfies conditions H1)-H3) (see (6.26)-(6.27)) and additionally it is lower semi-continuous with respect to the weak topology of $\left(L^{2}\left(\Omega, L^{\infty}(0, T ; \widetilde{W})\right)\right)^{2}$.

Theorem 8.1 Under above mentioned conditions the control problem $(\mathcal{P})$ admits, at least, one optimal solution

$$
(\widehat{U}, \widehat{Y}) \in \mathcal{U}_{a d}^{b} \times\left(L^{2}\left(\Omega, L^{\infty}(0, T ; \widetilde{W})\right) \cap L^{p}\left(\Omega, L^{\infty}(0, T ; V)\right)\right)
$$

where $\widehat{Y}$ is the unique solution of system (2.1) with $U=\widehat{U}$.

In addition, there exists a unique solution

$$
(\widehat{p}, \widehat{q}) \in L^{2}\left(\Omega, L^{\infty}(0, T ; W)\right) \times L^{2}\left(\Omega, L^{2}(0, T ; W)\right)
$$

of the backward stochastic differential equation

$$
\begin{cases}d \sigma(\widehat{p})=(-\nu \Delta \widehat{p}-\operatorname{curl} \sigma(\widehat{Y}) \times \widehat{p}+\operatorname{curl}(\sigma(\widehat{Y} \times \widehat{p}))+\nabla \pi-f) d t & \\ \operatorname{div} \widehat{p}=0 & -\left(\nabla_{y} L(t, \widehat{U}, \widehat{Y})+\nabla_{y} G(t, \widehat{Y})^{T} \widehat{q}\right) d t+\sigma(\widehat{q}) d W_{t}, \\ \widehat{p} \cdot \mathrm{n}=0, \quad(\mathrm{n} \cdot D \widehat{p}) \cdot \tau=0 & \text { in }(0, T) \times \mathcal{O}, \\ \widehat{p}(T)=h(\widehat{Y}(T)) & \text { on }(0, T) \times \Gamma,\end{cases}
$$

such that if $\widehat{Z}$ is the solution of system (5.2) for $Y=\widehat{Y}$ and $\Psi$ replaced by $\Psi-\widehat{U}$, then the duality property

$$
\mathbb{E} \int_{0}^{T}\left(\nabla_{y} L(t, \widehat{U}, \widehat{Y}), \widehat{Z}\right) d t+\mathbb{E}((\widehat{Z}(T)), \nabla h(\widehat{Y}(T)))_{V}=\mathbb{E} \int_{0}^{T}(\Psi-\widehat{U}, \widehat{p}) d t
$$

and the following optimality condition

$$
\mathbb{E} \int_{0}^{T}\left\{\left(\nabla_{u} L(t, \widehat{U}, \widehat{Y}), \Psi-\widehat{U}\right)+\int_{0}^{T}(\Psi-\widehat{U}, \widehat{p})\right\} d t \geq 0
$$

hold. 
Proof. 1st step: existence of an optimal control. Let us consider a minimizing sequence $\left\{\left(U_{n}, Y_{n}\right)\right\}$ of the functional $J$. Since the sequence $\left\{U_{n}\right\}$ belongs to $\mathcal{U}_{a d}^{b}$ which is a closed convex and bounded subset of $L^{p}\left(\Omega, L^{2}\left(0, T ; H^{1}(\mathcal{O})\right)\right), 4 \leq p<\infty$, then there exists a subsequence, still denoted by $\left\{U_{n}\right\}_{n=1}^{\infty}$, that converges weakly to $\widehat{U} \in L^{p}\left(\Omega, L^{2}\left(0, T ; H^{1}(\mathcal{O})\right)\right)$. As a consequence of Theorem 4.2 the sequence $\left\{Y_{n}\right\}$ is uniformly bounded in

$$
L^{2}\left(\Omega, L^{\infty}(0, T ; \widetilde{W})\right) \cap L^{p}\left(\Omega, L^{\infty}(0, T ; V)\right) .
$$

Therefore, there exists $\widehat{Y}$, such that

$$
Y_{n} \rightarrow \widehat{Y} \quad \text { weakly in } L^{2}\left(\Omega, L^{\infty}(0, T ; \widetilde{W})\right) \cap L^{p}\left(\Omega, L^{\infty}(0, T ; V)\right) .
$$

Using the same reasoning as in the proof of Theorem 4.2 (see the details in the article [9]), we may verify that $\widehat{Y}$ is solution of the stochastic differential system (2.1). On the other hand the functional $J$ is lower semi-continuous with respect to the weak topology of $\left(L^{2}\left(\Omega, L^{\infty}(0, T ; \widetilde{W})\right)\right)^{2}$

$$
J(\widehat{U}, \widehat{Y}) \leq \underline{\lim }_{n \rightarrow \infty} J\left(U_{n}, Y_{n}\right),
$$

which implies that $(\widehat{U}, \widehat{Y})$ is an optimal pair.

2nd step: Proof of the necessary optimality condition (8.50). Let $(\widehat{U}, \widehat{Y})$ be the optimal control pair. Let us consider arbitrary $\Psi \in \mathcal{U}_{a d}^{b}$. Then from Propositions 6.1 6.2 the Gâteaux derivative of the cost functional $J$ at the point $\widehat{U}$ in the direction $\Psi-\widehat{U}$ is given by

$$
\begin{aligned}
\left.\frac{d J(\widehat{U}+\rho(\Psi-\widehat{U}))}{d \rho}\right|_{\rho=0}= & \mathbb{E} \int_{0}^{T}\left\{\left(\nabla_{u} L(t, \widehat{U}, \widehat{Y}), \Psi-\widehat{U}\right)+\left(\nabla_{y} L(t, \widehat{U}, \widehat{Y}), \widehat{Z}\right)\right\} d t \\
& +\mathbb{E}(\nabla h(\widehat{Y}), \widehat{Z})_{V},
\end{aligned}
$$

where $\widehat{Z}$ is the Gâteaux derivative of the control to state map at point $\widehat{U}$ in the direction $\Psi-\widehat{U}$, being the unique solution of the stochastic linearized system (5.2) with $\Psi$ replaced by $\Psi-\widehat{U}$.

The set $\mathcal{U}_{a d}^{b}$ is convex, hence in the above calculation of $\frac{d J(\widehat{U}+\rho(\Psi-\widehat{U}))}{d \rho}$ we could take $\rho \in(0,1)$, that implies

$$
\mathbb{E} \int_{0}^{T}\left\{\left(\nabla_{u} L(t, \widehat{U}, \widehat{Y}), \Psi-\widehat{U}\right)+\left(\nabla_{y} L(t, \widehat{U}, \widehat{Y}), \widehat{Z}\right)\right\} d t+\mathbb{E}(\nabla h(\widehat{Y}(T)), \widehat{Z}(T))_{V} \geq 0 .
$$

Since here the duality property (7.44) can be written as (8.49), we easily verify that the solution $\widehat{p}$ of (8.48) fulfills the optimality condition (8.50).

Remark 8.2 Let us notice that a possible interpretation of the hypothesis (7.21) or (7.22) concerning the result obtained in Theorem 8.1 is the following:

a) In the case of "small" influence of the noise, i.e. L is taken small, then condition (7.21) holds, implying the controllability of the motion of the fluid for any viscosity values $\nu$;

b) In the case of "small" time interval [0,T], the condition (7.21) still holds, and we are able to control the fluid motion for any values of the viscosity and for any intensity of the noise;

c) For non axisymmetric domains, the "very" viscous fluids satisfy (7.22) and its motion can be controlled for any intensity of the noise and any interval of time. 


\section{Acknowledgment}

The work of N.V. Chemetov was supported by the Fundação para a Ciência e a Tecnologia (Portuguese Foundation for Science and Technology), project project UID/MAT/04561/2013.

The work of F. Cipriano was partially supported by the Fundação para a Ciência e a Tecnologia (Portuguese Foundation for Science and Technology) through the project UID/MAT/00297/2013 (Centro de Matemática e Aplicações).

\section{References}

[1] B. Andrews, C. Hopper, The Ricci Flow in Riemannian Geometry, 271, Lecture Notes in Mathematics, Springer-Verlag Berlin Heidelberg, 2011.

[2] N. Arada, F. Cipriano, Optimal control of non-stationary second grade fluids with Navierslip boundary conditions, arXiv:1511.01134 (2015) 1-30.

[3] N. Arada, F. Cipriano, Optimal control of steady second grade fluids with a Navier-slip boundary condition, arXiv:1511.00681 (2015) 1-31.

[4] H. I. BRECKNer, Approximation and optimal control of the stochastic Navier-Stokes equation. Ph.D. Thesis, Halle (Saale), 1999.

[5] A. V. Busuioc, D. Iftimie, A non-Newtonian fluid with Navier boundary conditions, J. Dynam. Diff. Eq. 18 (2006) 357-379.

[6] A. V. Busuioc, T. S. Ratiu, The second grade fluid and averaged Euler equations with Navier-slip boundary conditions, Nonlinearity 16 (2003) 1119-1149.

[7] N.V. Chemetov, F. Cipriano, Boundary layer problem: Navier-Stokes equations and Euler equations. Nonlinear Analysis: Real World Applications 14 (2013) 2091-2104

[8] N.V. Chemetov, F. Cipriano, The Inviscid Limit for the Navier-Stokes Equations with Slip Condition on Permeable Walls. J. Nonlinear Sci. 23 (2013) 731-750

[9] N.V. Chemetov, F. Cipriano, Well-posedness of stochastic second grade fluids. J. Math. Analysis and Applications (2017) DOI: 10.1016/j.jmaa.2017.04.060.

[10] D. Cioranescu, V. Girault, Weak and classical solutions of a family of second grade fluids, Int. J. Nonlinear Mech. 32 (1997) 317-335.

[11] D. Cioranescu, E. H. Ouazar, Existence and uniqueness for fluids of second grade, Nonlinear Partial Differential Equations and Their Applications (Collège de France Seminar, Paris, 1982/1983), 4 (Boston, MAPitman) (1984) 178-197.

[12] F. Cipriano, I. Torrecilla, Inviscid limit for $2 D$ stochastic Navier-Stokes equations. Journal of Stochastic Processes and their Applications, 125 (6) (2015) 2405-2426.

[13] T. Clopeau, A. Mikelic, R. Robert, On the vanishing viscosity limit for the $2 D$ incompressible Navier-Stokes equations with the friction type boundary conditions, Nonlinearity 11 (1998) 1625-1636.

[14] N. J. Cutland, K. Grzesiak, Optimal Control for Two-Dimensional Stochastic Navier-Stokes Equations, Appl. Math. Optim. 55 (2007) 61-91. 
[15] G. Da Prato, A. Debussche, Dynamic programming for the stochastic Navier-Stokes equations, Math. Modell. Numer. Anal. 34(2) (2000) 459-475.

[16] L. Desvillettes, C. Villani, On a variant of Korn's inequality arising in statistical mechanics, ESAIM Control Optim. Calc. Var. 8 (2002) 603-619.

[17] J. E. Dunn, R. L. FosDick, Thermodynamics, stability and boundedness of fluids of complexity 2 and fluids of second grade, Arch. Rational Mech. Anal. 56 (1974) 191-252.

[18] J. E. Dunn, K. R. Rajagopal, Fluids of differential type: Critical review and thermodynamical analysis, Int J. Eng. Sci. 33 (1995) 689-729.

[19] D. D. Holm, J. E. Marsden, R. S. Ratiu, Euler-Poincaré models of ideal fluids with nonlinear dissipation, Phys. Rev. Lett. 349 (1998) 4173-4177.

[20] D. D. Holm, J. E. Marsden, R. S. Ratiu, The Euler-Poincaré equations and semi-direct products with applications to continuum theories, Adv. Math. 137 (1998) 1-81.

[21] J. P. Kelliher, Navier-Stokes equations with Navier boundary conditions for a bounded domain in the plane, SIAM J. Math. Anal. 38 (2006) 210-232.

[22] LiseI, H., A minimum principle for the stochastic Navier-Stokes equation, Stud. Univ. Babeş-Bolyai Math. 45(2) (2000) 37-65.

[23] LISEI, H., Existence of optimal and Epsilon-optimal controls for the stochastic Navier-Stokes equation, Nonlinear Anal. Ser. A 51(1) (2002) 95-118.

[24] J. L. Menaldi, S. S. SRItharan, Impulse control of stochastic Navier-Stokes equations, Nonlinear Anal. 52(2) (2003) 357-381.

[25] W. Noll, C. Truesdell, The Nonlinear Field Theories of Mechanics, Encyclopedia of Physics, (ed. S. Flugge), Vol. III/3, Springer-Verlag, 1965.

[26] P. A. Razafimandimby, M. Sango, Strong solution for a stochastic model of twodimensional second grade fluids: Existence, uniqueness and asymptotic behavior, Nonlinear Analysis 75 (2012) 4251-4270.

[27] R. S. Rivlin, J. L. ERICKSEn, Stress-deformation relations for isotropic materials, Arch. Rational Mech. Anal. 4 (1955) 323-425.

[28] V. E. ŠČAdilov, V. A. Solonikov, On a boundary value problem for a stationary system of Navier-Stokes equations, Proc. Steklov Inst. Math. 125 (1973) 186-199.

[29] S.S. SRITharan, Deterministic and stochastic control of Navier-Stokes equation with linear, monotone, and hyperviscosities, Appl. Math. Optim. 41(2) (2000) 255-308. 